\title{
Distinct Landscapes of Genetic and Epigenetic Alterations of E2F Family Genes Between Esophageal Squamous Cell Carcinoma and Esophageal Adenocarcinoma
}

\section{Guo-Liang Zheng}

Cancer Hospital of China Medical University: Liaoning Cancer Institute and Hospital

Guo-Jun Zhang

Cancer Hospital of China Medical University: Liaoning Cancer Institute and Hospital

Tian-Yi Wu

Cancer Hospital of China Medical University: Liaoning Cancer Institute and Hospital

\section{Yan Zhao}

Cancer Hospital of China Medical University: Liaoning Cancer Institute and Hospital

\section{Zhi-Chao Zheng ( $\sim$ drzhengzc@126.com)}

Cancer Hospital of China Medical University: Liaoning Cancer Institute and Hospital https://orcid.org/0000-0002-5634-8853

\section{Research article}

Keywords: Esophageal cancer, esophageal squamous cell carcinoma, esophageal adenocarcinoma, E2F translation factors

Posted Date: November 20th, 2020

DOI: https://doi.org/10.21203/rs.3.rs-111352/v1

License: (c) (i) This work is licensed under a Creative Commons Attribution 4.0 International License. Read Full License 


\section{Abstract}

Background/Aims: Esophageal squamous cell carcinoma (ESCC) and esophageal adenocarcinoma (EAC) are primarily driven by different genetic changes. The E2F transcription factors (E2Fs) are involved in various malignancies; yet little is known about their roles in ESCC and EAC. This study aimed to investigate genetic and epigenetic alterations of the E2F family genes in ESCC and EAC, and to examine whether the differential changes are associated with the distinct mechanisms.

Materials and Methods: mRNA and methylation levels of E2F family genes in ESCC and EAC were retrieved from the database UALCAN. The cBioPortal for Cancer Genomics and Search Tool for the Retrieval of Interacting Genes (STRING) database were used to analyze genetic mutations and interaction networks for E2Fs, respectively.

Results: The genetic alteration patterns of E2Fs were different between ESCC and EAC. Furthermore, differences in methylation levels, CNV, and mutation frequency in certain E2F family genes were observed between ESCC and EAC. Moreover, E2Fs were co-expressed and interacted with proteins involved in the N6-methyladenosine (m6A) and histone modifications in EC. Additionally, Kaplan-Meier analysis revealed significant lower E2F1, E2F3, E2F5, E2F7, and E2F8 mRNA levels in association with better prognosis for EAC; while lower E2F1, E2F3, E2F6, E2F7, and E2F8 mRNA levels indicated poorer outcome for ESCC.

Conclusions: The different patterns of genetic and epigenetic alterations in the E2F family genes between ESCC and EAC are likely to be associated with different mechanisms. Certain E2Fs hold promise as biomarkers for differential diagnosis of EAC and ESCC, and for prognostic prediction.

\section{Introduction}

Esophageal cancer (EC) is the eighth most common cancer globally and one of the major causes of cancer-related death [1-3]. The histological subtypes of EC mainly include esophageal squamous cell carcinoma (ESCC) and esophageal adenocarcinoma (EAC) [4], of which ESCC is the most common histological form [5]. Unfortunately, the prognosis for patients with EC is poor, and this is mainly attributed to delays in the diagnosis and multidrug resistance [6]. In addition, different histological subtypes tend to occur in different parts of the esophagus and are likely to be driven by distinct genetic alterations through distinct pathogenic mechanisms, thus explaining, at least in part, the heterogeneity of EC [7]. Although substantial progress in diagnostic strategies and treatment techniques for EC has been made recently, the clinical outcomes of patients with EC remain unsatisfactory [8]. It has been documented that the two histological forms are likely to be driven by distinct genetic alterations via different mechanisms. Therefore, it is critical to better understand the genetic alterations and their relationships to the molecular mechanisms underlying the heterogeneity of the histological subtypes, especially ESCC and EAC, in order to guide the development of better diagnostic and therapeutic approaches. 
The E2F transcription factors (E2Fs) are a family of transcription factors consisting of activators (E2F1, $\mathrm{E} 2 \mathrm{~F} 2, \mathrm{E} 2 \mathrm{~F} 3 \mathrm{a}$, and E2F3b), repressors (E2F4 and E2F5), and inhibitors (E2F6, E2F7a, E2F7b, and E2F8); and they have been shown to be involved in the development, progression, and invasion of various malignancies [9-11]. Moreover, the E2F family members may have tumor-promoting or tumorsuppressive properties, depending mainly on the cancer cell type $[10,12,13]$. Previous studies have indicated that the E2F protein family members contain multiple highly conserved domains, including the DNA-binding domain, transactivation domain, and tumor suppressor protein-associated domain embedded within the transactivation domain $[9,14]$. Among the E2F family members, E2F1 is probably the most studied due to its regulatory mechanisms. However, until now, the roles of E2Fs in ESCC and EAC remain largely unknown.

In the present study, we aimed to investigate the genetic and epigenetic landscape alterations in the E2F family members between ESCC and EAC. The results gained through conducting this study may provide evidence for potentially novel roles of E2F family members in ESCC and EAC. In addition, they may help in the development of diagnostic, prognostic, and therapeutic approaches for EC.

\section{Materials And Methods}

Examination of mRNA expression and promoter region methylation of the E2F family genes in human ESCC and EAC

The mRNA expression and promoter region methylation levels of the E2F family genes [E2F1, E2F2, E2F3 (E2F3a and E2F3b), E2F4, E2F5, E2F6, E2F7 (E2F7a and E2F7b), and E2F8] in EC were analyzed via UALCAN (http://ualcan.path.uab.edu/index.html). The bioinformatics database UALCAN [15] is a comprehensive, user-friendly, and interactive online resource for analyzing cancer OMICS data, including The Cancer Genome Atlas (TCGA) datasets.

\section{Oncoplot of the E2F family genes in human ESCC and EAC}

The mutation frequency in the E2F family genes and the copy number in the esophageal carcinoma (ESCA) cohort of TCGA were assessed via the cBioPortal for Cancer Genomics (http://cbioportal.org)[16].

Coexpression network analysis of E2F family members and their incorporation with N6-methyladenosine (m6A)-modified proteins and histone-modified proteins in human ESCC and EAC

Coexpression network analysis was performed to assess the interactions between the E2F family members as well as their interactions with m6A-modified proteins and histone-modified proteins in human ESCC and EAC [17]. In the analysis, the University of California, Santa Cruz (UCSC) Xena browser (http://xena.ucsc.edu/), a visualization tool for analyzing and viewing public data hubs, was adopted to assess the expression levels of E2Fs in the ESCA cohort of TCGA based on the UCSC Toil RNA-seq Recompute dataset (TCGA and GTEx dataset). The RNA expression data of E2Fs (RSEM TPM, $n=19,131$ ) was downloaded as log2 $($ TPM +0.001$)$ values. The correlation analysis and visualization were 
implemented using the R-3.6 software (http://www.r-project.org/), including the ggcorrplot and cowplot packages. A P-value $<0.05$ indicated a statistically significant difference. Furthermore, the Search Tool for the Retrieval of Interacting Genes (STRING) (https://string-db.org/) database [18] was used to construct protein-protein interactions among E2Fs. In addition, the protein-protein interactions between E2Fs and m6A-related and histone-related proteins were constructed. Subsequently, how these proteins work together to promote the development of ESCA was examined.

Correlation analysis of E2F family members and resistance of anticancer drugs in human ESCC and EAC

The Gene Set Cancer Analysis [19] (GSCALite) (http://bioinfo.life.hust.edu.cn/web/GSCALite/) was used to assess the correlation of E2Fs and anticancer drug resistance. A Spearman correlation coefficient value $>0.20$ and a P-value $<0.05$ were set as the cut-off criteria.

\section{Survival analysis for E2Fs between human ESCC and EAC}

The prognostic value of E2Fs in human ESCC and EAC was analyzed using the Kaplan-Meier Plotter [20] (https://kmplot.com/analysis/), a bioinformatics database that integrates gene expression and clinical data.

\section{Results}

\section{mRNA expression profiles of E2F family member genes between ESCC and EAC}

Comparative analysis of the mRNA expression levels of E2F family members [E2F1, E2F2, E2F3 (E2F3a and E2F3b), E2F4, E2F5, E2F6, E2F7 (E2F7a and E2F7b), and E2F8] between EC (ESCC and EAC) versus the normal control as well as ESCC versus EAC were performed using the bioinformatics database UALCAN [15]. As shown in Fig. 1A, the mRNA expression levels of E2Fs were significantly increased in patients with ESCC or EAC, compared with the normal control $(P<0.05)$. Furthermore, the comparative analysis revealed that the mRNA expression levels of E2F4 and E2F7 were significantly higher in ESCC compared with EAC, while the mRNA expression levels of E2F5 and E2F8 were significantly lower than those in EAC $(P<0.05$, Fig. 1B), suggesting different patterns of E2F alterations between ESCC and EAC.

\section{Genetic and epigenetic alterations of E2Fs between human ESCC and EAC}

It has been well documented that genetic and epigenetic variations primarily drive gene expression variations. Therefore, comprehensive analysis of the epigenetic changes (e.g., methylation) and copy number variation (CNV) in the E2F family member genes between human ESCC and EAC were conducted using the bioinformatics database OUALCAN [15]. As shown in Fig. 2, the methylation levels in the gene promoter regions of E2F3, E2F4, and E2F8 were significantly greater in patients with ESCC or EAC, compared with the normal control; while the levels of E2F5 and E2F6 were significantly lower in ESCC or EAC, compared with the normal control $(P<0.05)$. Although there was a statistical difference in the methylation levels in the promoter regions of some E2F family member genes, it was noteworthy that the methylation levels in their promoter regions remained very low in human ESCC or EAC. These findings 
implied that the minimal changes of the methylation levels in the promoter regions of the E2F family member genes may not serve as a primary driver of gene expression variations.

In addition, comparative analysis of the CNVs in the E2F family member genes was performed using cBioPortal [16], and the resulting data are presented in Fig. 3. Amplification of E2F family member genes was frequently detected in ESCC as well as in EAC (Fig. 3A\&B).

Interactions between the E2F family members and the interplay with other proteins involved in m6A and histone modifications in human ESCC and EAC

It has been shown that an interaction between the E2F family members is associated with the development and progression of cancer $[9,10,21-24]$. Thus, the coexpression profiles of the E2F family member genes and their incorporation with protein-protein interactions in human ESCC and ECA as well as normal control tissues were investigated. It was found that the genes within the same functional class showed significantly correlated expression patterns in EC (Fig. 4A-C). Moreover, these E2Fs interacted with each other frequently in the protein-protein interaction networks, especially E2F1, based on the STRING database [18] (Fig. 4D). These findings suggest that cross-talk among the E2F family members may participate in the development and progression of EC.

E2Fs and enzymes in the m6A modification pathway showed a significant coexpression relationship (Fig. 5A) and an intermolecular regulatory relationship (Fig. 5B). In addition, E2Fs showed a significant coexpression relationship with enzymes involved in histone modulations (Fig. 6A) and an intermolecular regulatory relationship (Fig. 6B).

\section{Relationship between E2Fs and anticancer drug resistance}

Consistently, it has been widely accepted that genetic alterations affect the sensitivity of patients to anticancer drugs as well as the patient prognosis $[25,26]$. Therefore, the correlation between the mRNA expression of E2Fs and the sensitivity to anticancer drugs based on the Genomics of Drug Sensitivity in Cancer dataset [27]was examined via the bioinformatics database GSCALite [19] (Fig. 7).

\section{Correlation between the MRNA expression patterns and the prognosis of patients with ESCC or EAC}

The effects of the mRNA expression of E2Fs on the survival of patients with ESCC or EAC were further assessed. Kaplan-Meier Plotter analysis revealed a correlation between the mRNA levels of E2Fs and the survival of patients with ESCC or EAC. Notably, lower E2F1, E2F3, E2F5, E2F7, and E2F8 mRNA expression levels predicted a better prognosis for EAC (Fig. 8A); whereas lower E2F1, E2F3, E2F6, E2F7, and E2F8 mRNA expression levels were associated with poorer clinical outcomes for ESCC (Fig. 8B).

\section{Discussion}

In this study, for the first time, the expression and significance of the E2F family members between the two main histological subtypes of EC (ESCC and EAC) were systemically analyzed. The novel findings of the present study were as follows: (1) The mRNA expression levels of the E2F family members were 
significantly elevated in EC compared with the normal control tissues. (2) The patterns of the E2F gene expression alterations were different between the two EC forms: ESCC and EAC. (3) The genetic mutations (e.g., mutation frequency, CNVs) and epigenetic changes (e.g., methylation and histone modifications) in some E2F family member genes were different between ESCC and EAC. (4) The coexpression profiles of E2F family member genes and their incorporation with protein-protein interactions revealed interactions between the E2F family members and interplay with proteins involved in m6A and histone modifications in EC. (5) Kaplan-Meier analysis showed a significant correlation of lower levels of E2F1, E2F3, E2F5, E2F7, and E2F8 mRNA expression with a better prognosis for EAC; and lower E2F1, E2F3, E2F6, E2F7, and E2F8 mRNA levels indicated a poorer outcome for ESCC. Thus, these E2Fs have potential as biomarkers in the differential diagnosis of EAC and ESCC as well as in the prognosis of EC.

The abnormal expression of E2F family member genes has been detected in a range of malignancies. For instance, overexpression of E2F1 has been shown to contribute to the development of EC, and this effect is enhanced by dysregulated pRb-p53-MDM2 circuitry [28].In addition, amplification of E2F1 is frequently found in ESCC [29]. In our study, TCGA datasets revealed that the expression of E2F1 was higher in human EC than in normal tissues. However, E2F1 mRNA expression was not significantly altered between the patients with ESCC versus those with EAC. Using the Kaplan-Meier Plotter, we found that the upregulation of E2F1 mRNA expression was significantly associated with a good prognosis (overall survival, OS) in ESCC, while downregulation of E2F2 mRNA expression predicted a good prognosis (OS) in EAC.

E2F2 is a tumor activator in many types of cancer. Using a high-frequency subpathway mining approach, $\mathrm{Li}$ et al. identified a six-gene model containing E2F2 that predicted clinical outcomes in patients with ESCC [30]. In the present study, the mRNA expression of E2F2 was not significantly different between ESCC and EAC. Furthermore, the mRNA expression of E2F2 was not significantly associated with the OS in either ESCC or EAC.

The abnormal overexpression of E2F3 is thought to be an oncogenic event in patients with EC. A novel four-gene signature including E2F3 was identified, externally validated, and shown to provide excellent distinction between EAC and the condition of Barrett's esophagus [31]. In this study, we demonstrated that the expression of E2F3 in EC tissues was higher than that in normal tissues. Additionally, the mRNA expression of E2F3 was significantly different between ESCC and EAC. Surprisingly, greater levels of E2F3 mRNA expression were significantly associated with a higher OS in the patients with ESCC, while lower levels of E2F3 mRNA expression predicted a higher OS in the patients with EAC.

E2F4 has been reported to be associated with the occurrence and progression of malignant tumors [32]. In general, it has been thought that E2F4 may play an important role in inhibiting proliferation-related genes. For example, Bultmann et al. have identified E2F4 as a transcription repressor that strongly regulates the expression of latent transforming growth factor-beta-binding protein $4 \mathrm{~S}$ to inhibit the migration of esophageal cancer cells [33]. In the present study, the expression of E2F4 was demonstrated 
to be upregulated in EC tissues compared with normal tissues. Interestingly, the expression of E2F4 mRNA was significantly different between ESCC and EAC. Furthermore, greater levels of E2F4 mRNA expression predicted a poorer OS in the patients with ESCC using the Kaplan-Meier Plotter. Nevertheless, it must be pointed out that this finding seems to be inconsistent with the role of E2F4 as a tumor suppressor.

E2F5 has been found to be highly expressed in several malignancies, including glioblastoma and prostate cancer. However, the expression and prognostic effects of E2F5 on EC have been rarely reported. Sun et al. have found that microRNA-544 inhibited ESCC cell proliferation and enhanced the sensitivity to cisplatin by repressing E2F5 [34]. In this study, it was shown that the expression of E2F5 in EC tissues was higher than that in normal tissues. In addition, higher E2F5 expression was significantly correlated with a better OS in the patients with ESCC.

The expression and prognostic effects of E2F6 in ESCA have not been reported previously. Li et al.have found that miR-424 can inhibit the proliferation and migration abilities of A549 cells by negatively regulating the expression of E2F6 in non-small cell lung cancer (NSCLC) [35]. E2F6 is also upregulated in NSCLC blood samples, indicating that E2F6 possesses an oncogenic feature in lung cancer [36]. In this study, it was demonstrated that the expression of E2F6 in ESCC tissues was higher than that in normal tissues. Moreover, a higher E2F6 expression level was significantly correlated with a poorer OS in the patients with EAC.

E2F7 and E2F8 are recently identified, novel members of the E2F family. Considering that a difference in the tumor histological subtypes could influence the measured tumor gene expression, they also likely serve as activators. E2F7 and E2F8 have been shown to be activators of transcription, while E2F8 binds to and activates the cyclin D1 promoter in a dominantly negative manner by blocking other E2Fs. E2F7 and E2F8 directly bind to and stimulate the promoter of vascular endothelial growth factor $A$ by cooperating with hypoxia-inducible factor-1. To date, the roles of E2F7 and E2F8 in the development and progression of EC remain unclear. E2F8 has been reported to be upregulated in ESCC, thus promoting cell proliferation and affecting the expression of cyclin D1/p21 [37]. In the present study, E2F7 and E2F8 were significantly overexpressed in ESCC and EAC tissues, and their expression levels were markedly different between ESCC and EAC. Interestingly, higher E2F7 and E2F8 expression levels were significantly correlated with a better OS in the patients with ESCC, while lower levels of E2F7 and E2F8 mRNA expression predicted a better OS in the patients with EAC.

Taken together, these novel findings reveal distinct landscapes of genetic and epigenetic alterations in the E2F family genes between ESCC and EAC, which may contribute to different molecular mechanisms for the two histological forms of EC through interactions among the E2F family members or with other functional proteins. This study may have important clinical implications that certain E2Fs hold promise as biomarkers for the differential diagnosis of EAC and ESCC, or in the prognosis of patients with EC.

\section{Abbreviations}


$\mathrm{EAC}=$ Esophageal Adenocarcinoma; $\mathrm{ESCA}=$ Esophageal Carcinoma; $\mathrm{ESCC}=$ Esophageal Squamous Cell Carcinoma; NSCLC $=$ Non-Small Cell Lung Cancer; OS = Overall Survival; STRING $=$ Search Tool for The Retrieval of Interacting Genes; TCGA = The Cancer Genome Atlas; UCSC = The University of California, Santa Cruz.

\section{Declarations}

\section{Acknowledgments}

We would like to thank Prof. Li-Zhao Wu from the Department of Pathophysiology, China Medical University, for his guidance on E2Fs.

\section{Availability of data and material}

All data generated or analyzed during this study are included in this published article.

\section{Author Contributions}

ZGL made substantial contributions to the conception and design of the study. ZGJ and WTY conducted data acquisition, analysis, and interpretation. ZY and ZZC drafted and revised the manuscript. All authors agree to be accountable for all aspects of the work in ensuring that questions related to the accuracy or integrity have been appropriately resolved. All authors have read and approved the final manuscript.

\section{Ethics approval and consent to participate}

The data were downloaded from public databases, and there are no ethical concerns regarding the data. Ethical approval was not applicable in this study.

\section{Patient consent for publication}

Not applicable.

\section{Competing interests}

The authors declare that there are no conflicts of interest related to this study.

\section{References}

1. Torre LA, Bray F, Siegel RL, Ferlay J, Lortet-Tieulent J, Jemal A. Global cancer statistics, 2012. CA Cancer J Clin. 2015;65:87-108.

2. Burstein HJ, Krilov L, Aragon-Ching JB, Baxter NN, Chiorean EG, Chow WA, De Groot JF, Devine SM, DuBois SG, El-Deiry WS, Epstein AS, Heymach J, Jones JA, Mayer DK, Miksad RA, Pennell NA, Sabel MS, Schilsky RL, Schuchter LM, Tung N, Winkfield KM, Wirth LJ, Dizon DS. Clinical Cancer Advances 
2017: Annual Report on Progress Against Cancer From the American Society of Clinical Oncology. J Clin Oncol. 2017;35:1341-67.

3. Bray F, Ferlay J, Soerjomataram I, Siegel RL, Torre LA, Jemal A. Global cancer statistics 2018: GLOBOCAN estimates of incidence and mortality worldwide for 36 cancers in 185 countries. CA Cancer J Clin. 2018;68:394-424.

4. Ohashi S, Miyamoto S, Kikuchi O, Goto T, Amanuma Y, Muto M. Recent Advances From Basic and Clinical Studies of Esophageal Squamous Cell Carcinoma. Gastroenterology. 2015;149:1700-15.

5. Pennathur A, Gibson MK, Jobe BA, Luketich JD. Oesophageal carcinoma. Lancet. 2013;381:400-12.

6. Wang C, Wang J, Chen Z, Gao Y, He J. Immunohistochemical prognostic markers of esophageal squamous cell carcinoma: a systematic review. Chin J Cancer. 2017;36:65.

7. Bollschweiler E, Metzger R, Drebber U, Baldus S, Vallböhmer D, Kocher M, Hölscher AH. Histological type of esophageal cancer might affect response to neo-adjuvant radiochemotherapy and subsequent prognosis. Ann Oncol. 2009;20:231-8.

8. Njei B, McCarty TR, Birk JW. Trends in esophageal cancer survival in United States adults from 1973 to 2009: A SEER database analysis. J Gastroenterol Hepatol. 2016;31:1141-6.

9. DeGregori J, Johnson DG. Distinct and Overlapping Roles for E2F Family Members in Transcription, Proliferation and Apoptosis. Curr Mol Med. 2006;6:739-48.

10. Johnson DG, Degregori J. Putting the Oncogenic and Tumor Suppressive Activities of E2F into Context. Curr Mol Med. 2006;6:731-8.

11. Chen HZ, Tsai SY, Leone G. Emerging roles of E2Fs in cancer: an exit from cell cycle control. Nat Rev Cancer. 2009;9:785-97.

12. Alonso MM, Alemany R, Fueyo J, Gomez-Manzano C. E2F1 in gliomas: a paradigm of oncogene addiction. Cancer Lett. 2008;263:157-63.

13. Crosby ME, Almasan A. Opposing roles of E2Fs in cell proliferation and death. Cancer Biol Ther. 2004;3:1208-11.

14. Bandara LR, Buck VM, Zamanian M, Johnston LH, La Thangue NB. Functional synergy between DP-1 and E2F-1 in the cell cycle-regulating transcription factor DRTF1/E2F. EMBO J. 1993;12:4317-24.

15. Chandrashekar DS, Bashel B, Balasubramanya S, Creighton CJ, Ponce-Rodriguez I, Chakravarthi B, Varambally S. UALCAN: A Portal for Facilitating Tumor Subgroup Gene Expression and Survival Analyses. Neoplasia. 2017;19:649-58.

16. Gao J, Aksoy BA, Dogrusoz U, Dresdner G, Gross B, Sumer SO, Sun Y, Jacobsen A, Sinha R, Larsson E, Cerami E, Sander C, Schultz N. Integrative analysis of complex cancer genomics and clinical profiles using the cBioPortal. Sci Signal. 2013;6:pl1.

17. Tay Y, Kats L, Salmena L, Weiss D, Tan SM, Ala U, Karreth F, Poliseno L, Provero P, Di Cunto F, Lieberman J, Rigoutsos I, Pandolfi PP. Coding-independent regulation of the tumor suppressor PTEN by competing endogenous mRNAs. Cell. 2011;147:344-57. 
18. Szklarczyk D, Franceschini A, Wyder S, Forslund K, Heller D, Huerta-Cepas J, Simonovic M, Roth A, Santos A, Tsafou KP, Kuhn M, Bork P, Jensen LJ, von Mering C. STRING v10: protein-protein interaction networks, integrated over the tree of life. Nucleic Acids Res. 2015;43:D447-52.

19. Liu CJ, Hu FF, Xia MX, Han L, Zhang Q, Guo AY. GSCALite: a web server for gene set cancer analysis. Bioinformatics. 2018;34:3771-2.

20. Nagy Á, Lánczky A, Menyhárt O, Győrffy B. Validation of miRNA prognostic power in hepatocellular carcinoma using expression data of independent datasets. Sci Rep. 2018;8:9227.

21. Yang WW, Shu B, Zhu Y, Yang HT. E2F6 inhibits cobalt chloride-mimetic hypoxia-induced apoptosis through E2F1. Mol Biol Cell. 2008;19:3691-700.

22. Zalmas LP, Zhao X, Graham AL, Fisher R, Reilly C, Coutts AS, La Thangue NB. DNA-damage response control of E2F7 and E2F8. EMBO Rep. 2008;9:252-9.

23. Liu B, Shats I, Angus SP, Gatza ML, Nevins JR. Interaction of E2F7 transcription factor with E2F1 and C-terminal-binding protein (CtBP) provides a mechanism for E2F7-dependent transcription repression. J Biol Chem. 2013;288:24581-9.

24. Moon NS, Dyson N. E2F7 and E2F8 keep the E2F family in balance. Dev Cell. 2008;14:1-3.

25. Sonnemann J, Gänge J, Pilz S, Stötzer C, Ohlinger R, Belau A, Lorenz G, Beck JF. Comparative evaluation of the treatment efficacy of suberoylanilide hydroxamic acid (SAHA) and paclitaxel in ovarian cancer cell lines and primary ovarian cancer cells from patients. BMC Cancer. 2006;6:183.

26. Zhu H, Luo H, Zhang W, Shen Z, Hu X, Zhu X. Molecular mechanisms of cisplatin resistance in cervical cancer. Drug Des Devel Ther. 2016;10:1885-95.

27. Yang W, Soares J, Greninger P, Edelman EJ, Lightfoot H, Forbes S, Bindal N, Beare D, Smith JA, Thompson IR, Ramaswamy S, Futreal PA, Haber DA, Stratton MR, Benes C, McDermott U, Garnett MJ. Genomics of Drug Sensitivity in Cancer (GDSC): a resource for therapeutic biomarker discovery in cancer cells. Nucleic Acids Res. 2013;41:D955-61.

28. Itoshima T, Fujiwara T, Waku T, Shao J, Kataoka M, Yarbrough WG, Liu TJ, Roth JA, Tanaka N, Kodama M. Induction of apoptosis in human esophageal cancer cells by sequential transfer of the wild-type p53 and E2F-1 genes: involvement of p53 accumulation via ARF-mediated MDM2 downregulation. Clin Cancer Res. 2000;6:2851-9.

29. Fujita Y, Sakakura C, Shimomura K, Nakanishi M, Yasuoka R, Aragane H, Hagiwara A, Abe T, Inazawa $\mathrm{J}$, Yamagishi H. Chromosome arm 20q gains and other genomic alterations in esophageal squamous cell carcinoma, as analyzed by comparative genomic hybridization and fluorescence in situ hybridization. Hepatogastroenterology. 2003;50:1857-63.

30. Li M, Zhao J, Li X, Chen Y, Feng C, Qian F, Liu Y, Zhang J, He J, Ai B, Ning Z, Liu W, Bai X, Han X, Wu Z, Xu X, Tang Z, Pan Q, Xu L, Li C, Wang Q, Li E. HiFreSP: A novel high-frequency sub-pathway mining approach to identify robust prognostic gene signatures. Brief Bioinform. 2019.

31. Maag J, Fisher OM, Levert-Mignon A, Kaczorowski DC, Thomas ML, Hussey DJ, Watson DI, Wettstein A, Bobryshev YV, Edwards M, Dinger ME, Lord RV. Novel Aberrations Uncovered in Barrett's 
Esophagus and Esophageal Adenocarcinoma Using Whole Transcriptome Sequencing. Mol Cancer Res. 2017;15:1558-69.

32. Lee BK, Bhinge AA, lyer VR. Wide-ranging functions of E2F4 in transcriptional activation and repression revealed by genome-wide analysis. Nucleic Acids Res. 2011;39:3558-73.

33. Bultmann I, Conradi A, Kretschmer C, Sterner-Kock A. Latent transforming growth factor $\beta$-binding protein 4 is downregulated in esophageal cancer via promoter methylation. PLoS One. 2013;8:e65614.

34. Sun F, Zhang C, Ma D, Wang K. MicroRNA-544 inhibits esophageal squamous cell carcinoma cell proliferation and enhances sensitivity to cisplatin by repressing E2F transcription factor 5 . Oncol Lett. 2019;18:4203-9.

35. Li H, Lan H, Zhang M, An N, Yu R, He Y, Gan C. [Effects of miR-424 on Proliferation and Migration Abilities in Non-small Cell Lung Cancer A549 Cells and Its Molecular Mechanism]. Zhongguo Fei Ai Za Zhi. 2016;19:571-6.

36. Barh D, Jain N, Tiwari S, Field JK, Padin-Iruegas E, Ruibal A, López R, Herranz M, Bhattacharya A, Juneja L, Viero C, Silva A, Miyoshi A, Kumar A, Blum K, Azevedo V, Ghosh P, Liloglou T. A novel in silico reverse-transcriptomics-based identification and blood-based validation of a panel of sub-type specific biomarkers in lung cancer. BMC Genomics. 2013;14 Suppl 6:S5.

37. Chang $\mathrm{H}$, Song J, Wu J, Zhang Y. E2F transcription factor 8 promotes cell proliferation via CCND1/p21 in esophageal squamous cell carcinoma. Onco Targets Ther. 2018;11:8165-73.

\section{Figures}



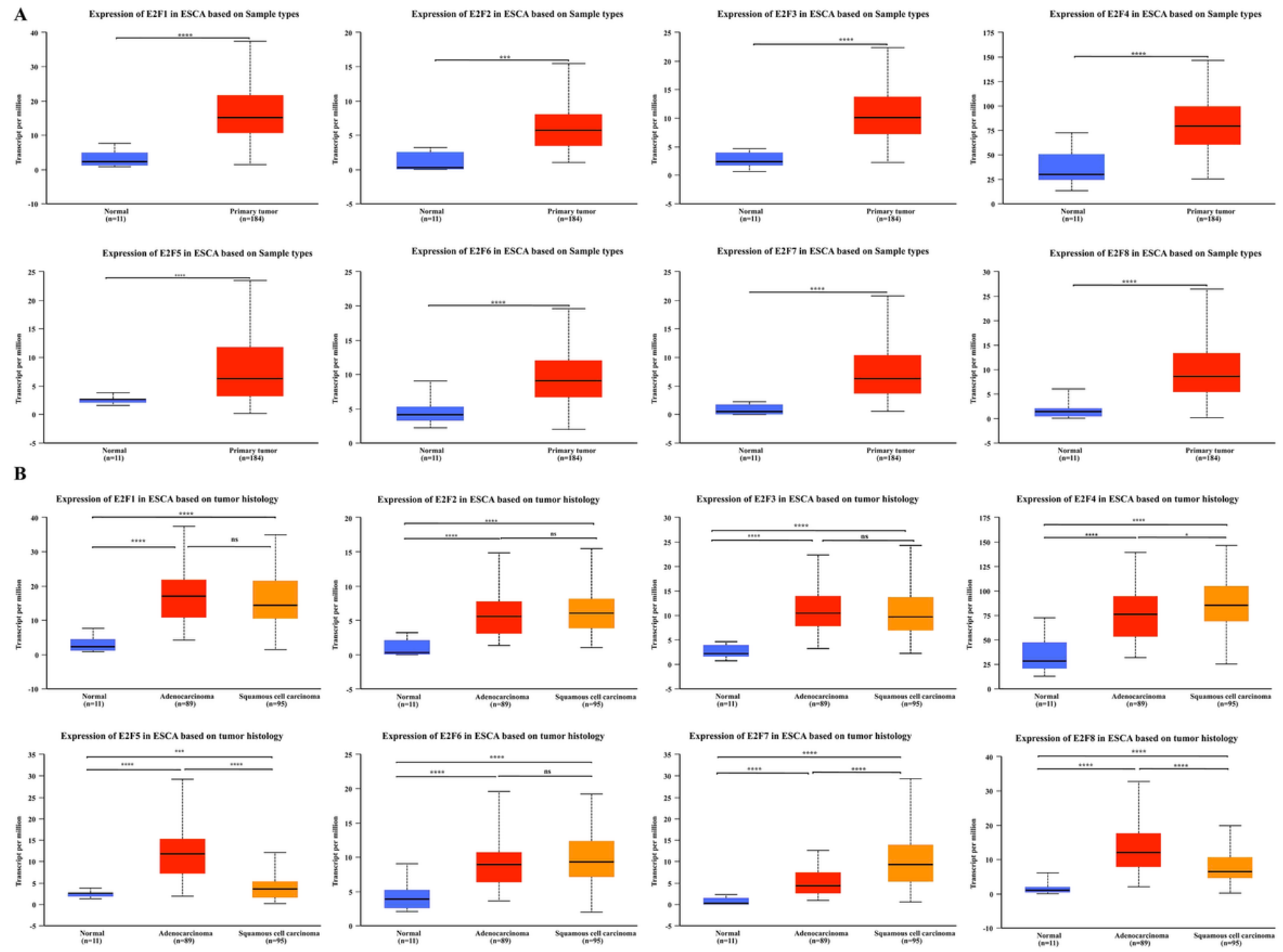

Figure 1

The mRNA expression levels of E2F family member genes in ESCC, ECA, and normal control tissues. (A) The mRNA expression levels in normal control and ESCA tissues. (B) The mRNA expression levels in the two different histological subtypes of ESCA (ESCC and ECA). ESCA: esophageal carcinoma; ESCC: esophageal squamouscell carcinoma; EAC: esophageal adenocarcinoma. *, statistically significant

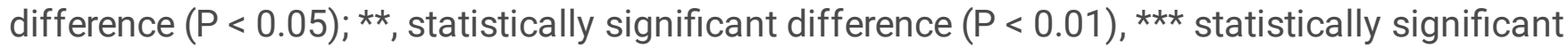
difference $(P<0.001)$; $* \star \star \star$, statistically significant difference $(P<0.0001)$. 

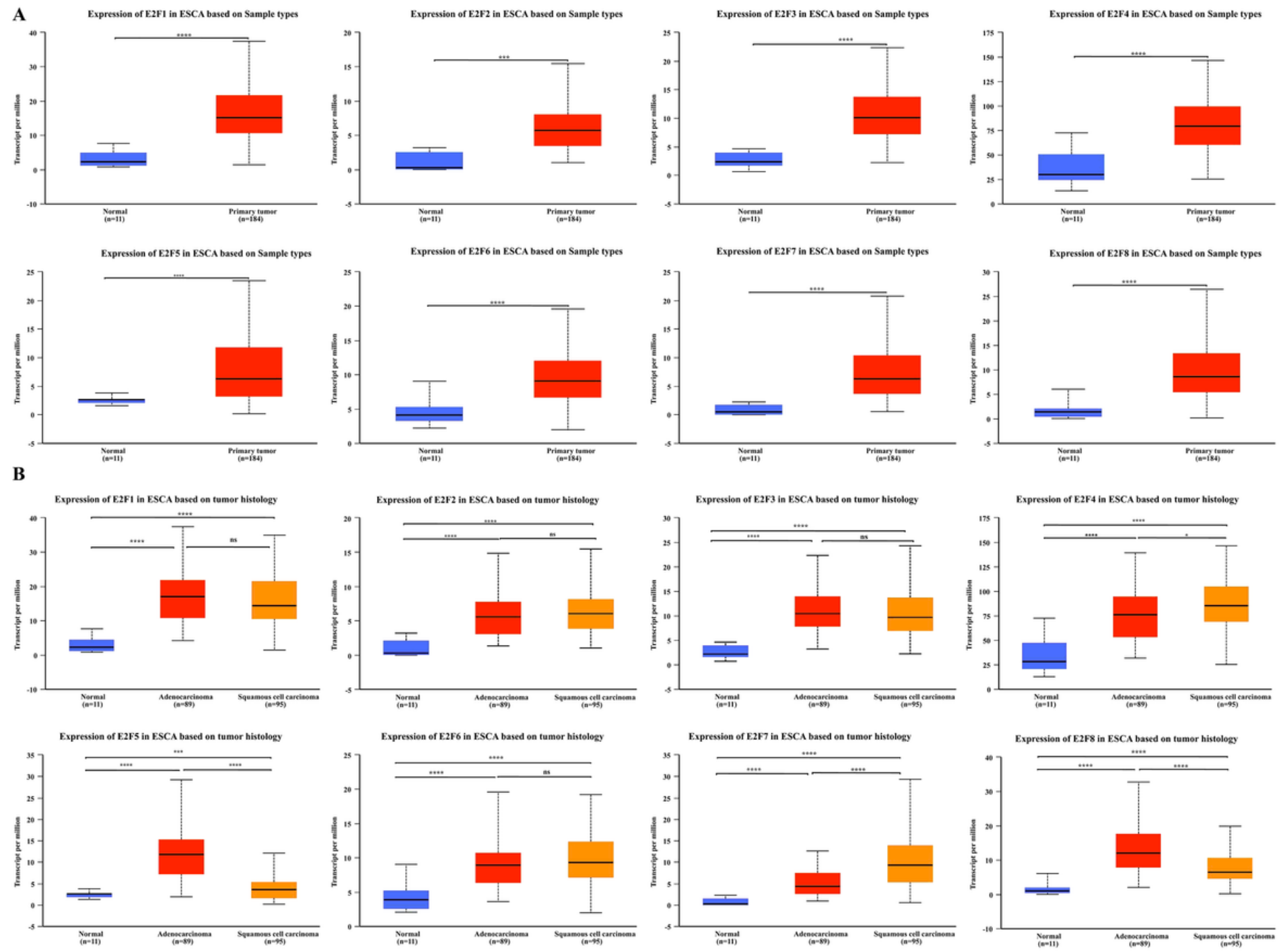

Figure 1

The mRNA expression levels of E2F family member genes in ESCC, ECA, and normal control tissues. (A) The mRNA expression levels in normal control and ESCA tissues. (B) The mRNA expression levels in the two different histological subtypes of ESCA (ESCC and ECA). ESCA: esophageal carcinoma; ESCC: esophageal squamouscell carcinoma; EAC: esophageal adenocarcinoma. *, statistically significant

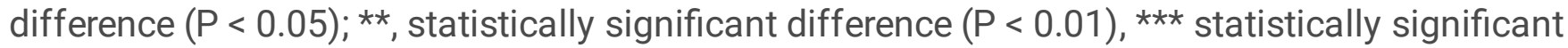
difference $(P<0.001)$; $\star \star \star \star$, statistically significant difference $(P<0.0001)$. 

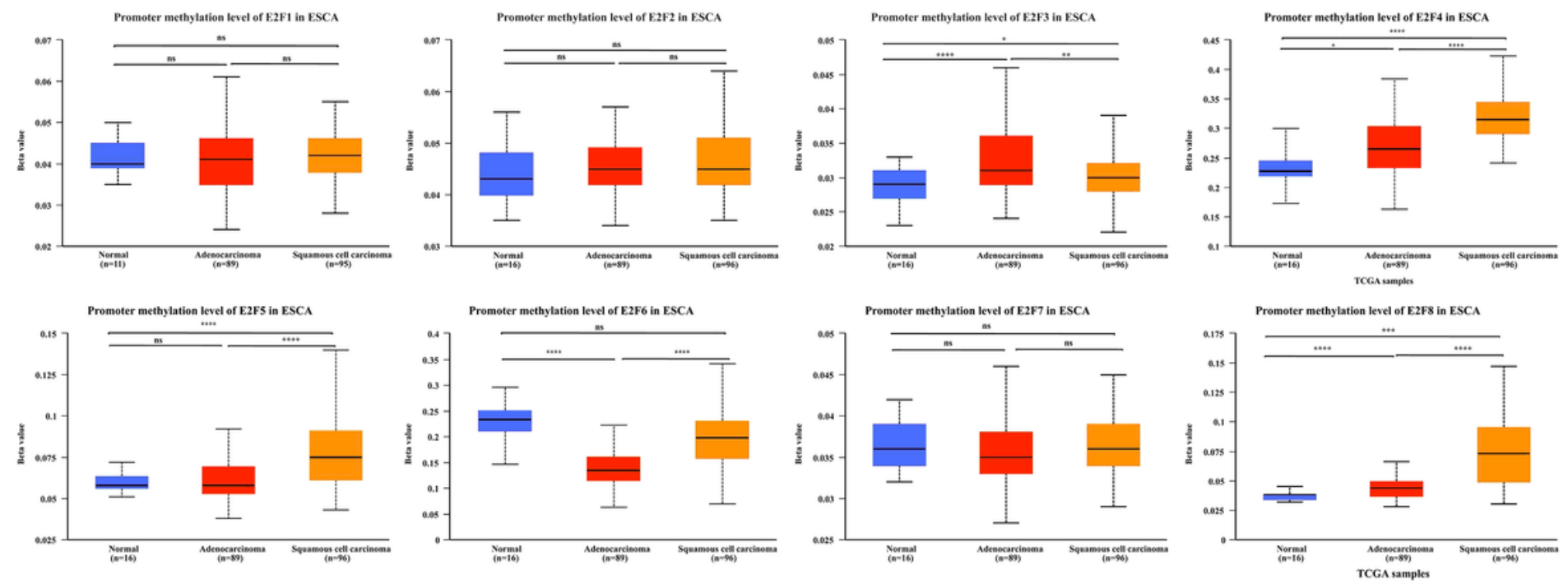

\section{Figure 2}

Methylation levels in the promoter regions of E2F family member genes in the different histological subtypes of ESCA. ESCA: esophageal carcinoma; ESCC: esophageal squamous cell carcinoma; EAC: esophageal adenocarcinoma. The beta value indicates the level of DNA methylation ranging from 0 (unmethylated) to 1 (fully methylated). Different beta value cut-offs indicated hypermethylation (beta value: $0.7-0.5$ ) or hypomethylation (beta value: $0.3-0.25$ ). The above boxplot represents the beta values of CpG probes located up to 1500 bp upstream of the gene's start site (TSS200, TSS1500). ns, nonsignificant difference $(P>0.05)$; *, statistically significant difference $(P<0.05)$; **, statistically significant difference $(P<0.01)$, *** statistically significant difference $(P<0.001)$; ${ }^{\star \star \star *}$, statistically significant difference $(P<0.0001)$.
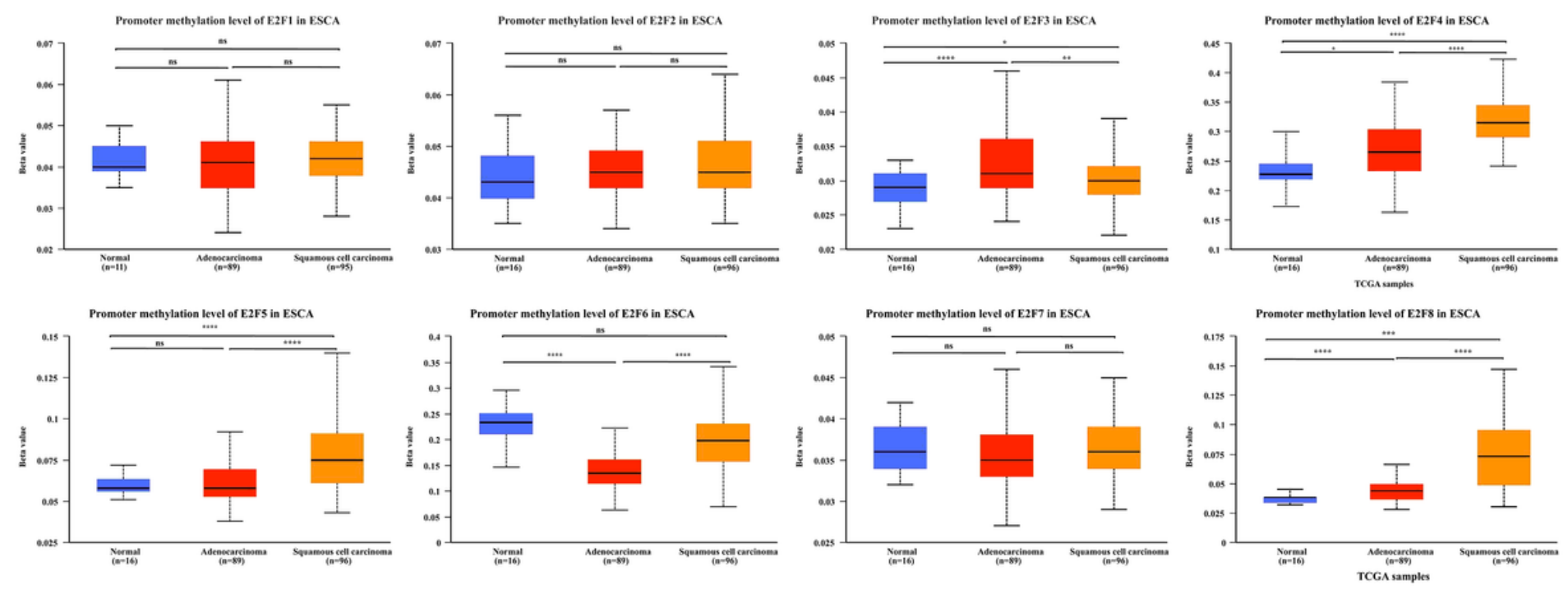

Figure 2

Methylation levels in the promoter regions of E2F family member genes in the different histological subtypes of ESCA. ESCA: esophageal carcinoma; ESCC: esophageal squamous cell carcinoma; EAC: 
esophageal adenocarcinoma. The beta value indicates the level of DNA methylation ranging from 0 (unmethylated) to 1 (fully methylated). Different beta value cut-offs indicated hypermethylation (beta value: $0.7-0.5$ ) or hypomethylation (beta value: $0.3-0.25$ ). The above boxplot represents the beta values of CpG probes located up to 1500 bp upstream of the gene's start site (TSS200, TSS1500). ns, nonsignificant difference $(P>0.05)$; *, statistically significant difference $(P<0.05)$; **, statistically significant difference $(P<0.01)$, *** statistically significant difference $(P<0.001)$; ${ }^{\star \star \star \star}$, statistically significant difference $(P<0.0001)$.

\section{A}

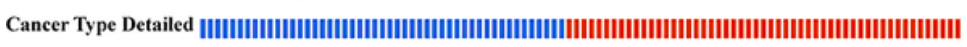

E2F1

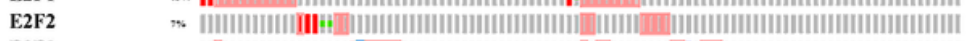

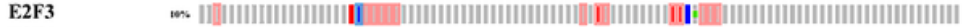

E2F4

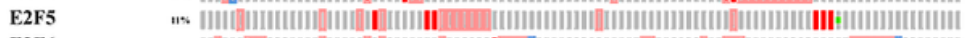

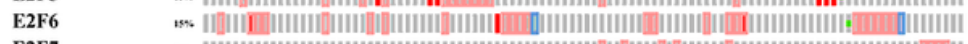

E2F7 $\quad$ "

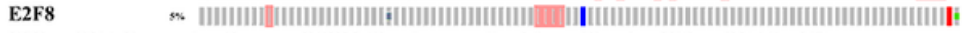

E2Fs mRNA Expression Zscores, RSEM (Batch normalized from Illumina HiSeq_RNASeqV2)

E2F1

E2F3

$\mathrm{E} 2 \mathrm{~F} 4$
$\mathrm{E} 2 \mathrm{~F} 5$

E2F5
E2F6

E2F8

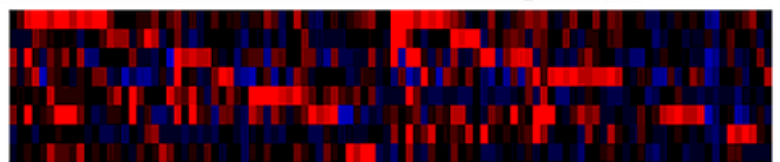

Genetic Alteration

i Missense Mutation (unknown significance) i Truncating Mutation (unknown signifieance)

| Amplification Deep Deletion ImRNA High

ImRNA Low INo alterations

Cancer Type Detailed I Esophageal Adenocarcinoma IEsophageal Squamous Cell Careinoma

Expression Heatmap $\quad-3=3$

B

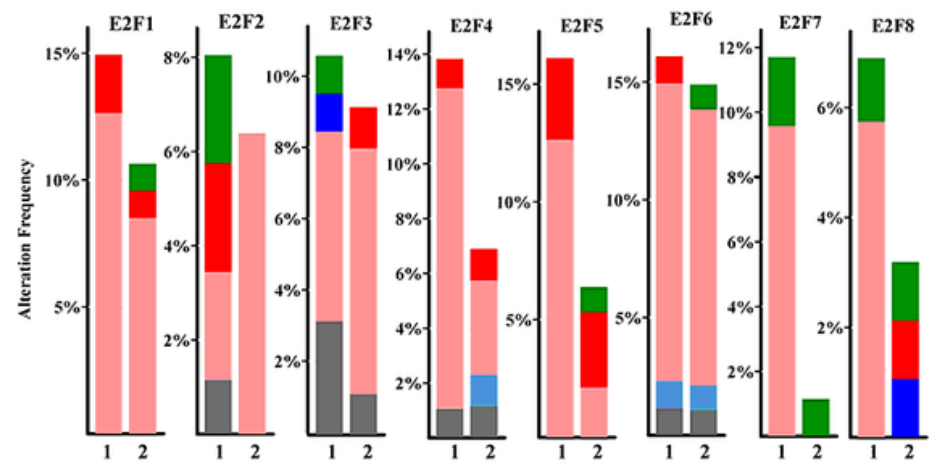

Mutation data + + Mutation

CNA data + + + Amplification $\bullet$ Deep Deletion 2 Esophageal Squamous Cell Carcinoma mRNA data + + $\circ$ mRNA High $\odot$ mRNAL Low

- Multiple Alterations

\section{Figure 3}

Analysis of copy number variations in the E2F family member genes in ESCC, ECA, and normal control tissues. cBioPortal for Cancer Genomics identifying oncoplot of the E2F genes commonly mutated in the TCGA ESCA cohort in ESCC and EAC. ESCA: esophageal carcinoma; ESCC: esophageal squamous cell carcinoma; EAC: esophageal adenocarcinoma.

A

\section{E2Fs transcription factor family genetic alteration}

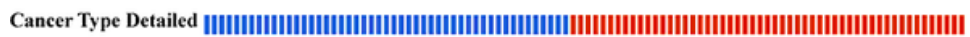

E2F1

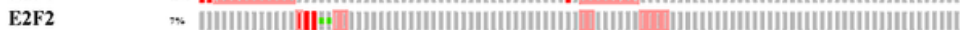

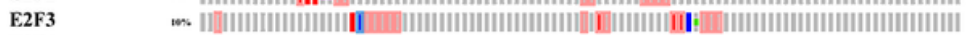

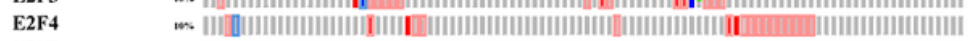

E2F5 2 "

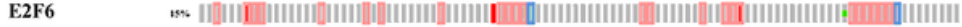

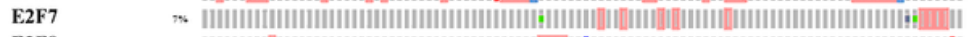

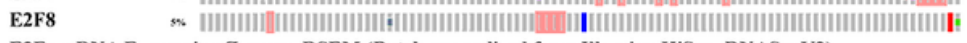

E2Fs mRNA Expression Zscores, RSEM (Batch normalized from Illumina HiSeq_RNASeqV2)

E2F1

E2F2

$\mathrm{E} 2 \mathrm{~F} 4$
$\mathrm{E} 2 \mathrm{~F} 5$

E2F5

E2F7
E2F8

Genetic Alteration

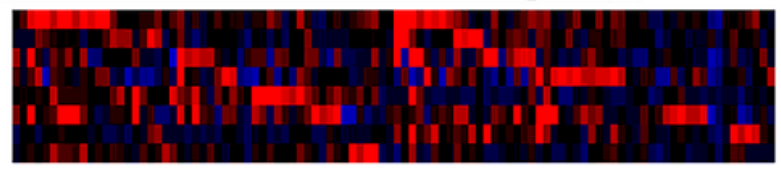

i Missense Mutation (unknown significance) i Truncating Mutation (unknown significance)

| Amplification | Imeep Deletion

\mRNA Low INo alterations

Cancer Type Detailed | Esophageal Adenocarcinoma | Esophageal Squamous Cell Carcinoma

Expression Heatmap $\quad-3 \square=3$
B

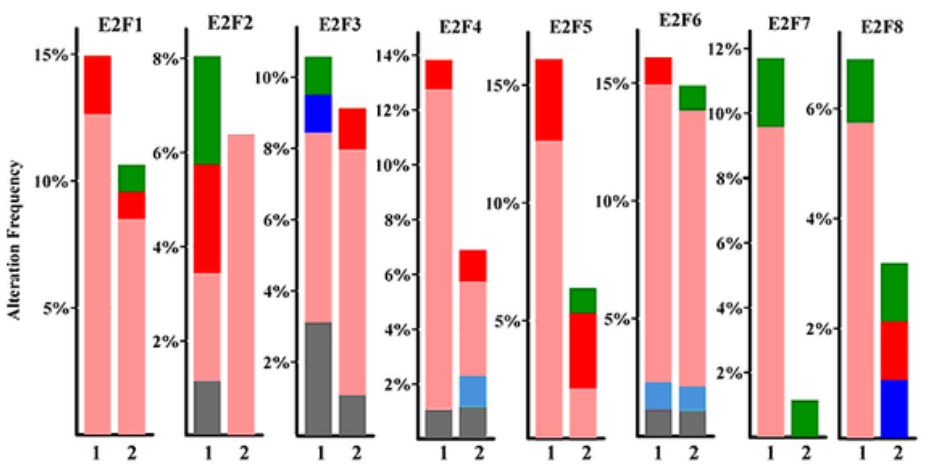

Mutation data $++\circ$ Mutation

1 Esophageal Adenocarcinoma

2 Esophageal Squamous Cell Carcinoma

mRNA data + + $\circ$ mRNA High $\odot$ mRNA Low

- Multiple Alterations

Figure 3 
Analysis of copy number variations in the E2F family member genes in ESCC, ECA, and normal control tissues. cBioPortal for Cancer Genomics identifying oncoplot of the E2F genes commonly mutated in the TCGA ESCA cohort in ESCC and EAC. ESCA: esophageal carcinoma; ESCC: esophageal squamous cell carcinoma; EAC: esophageal adenocarcinoma.

A

\section{EAC}
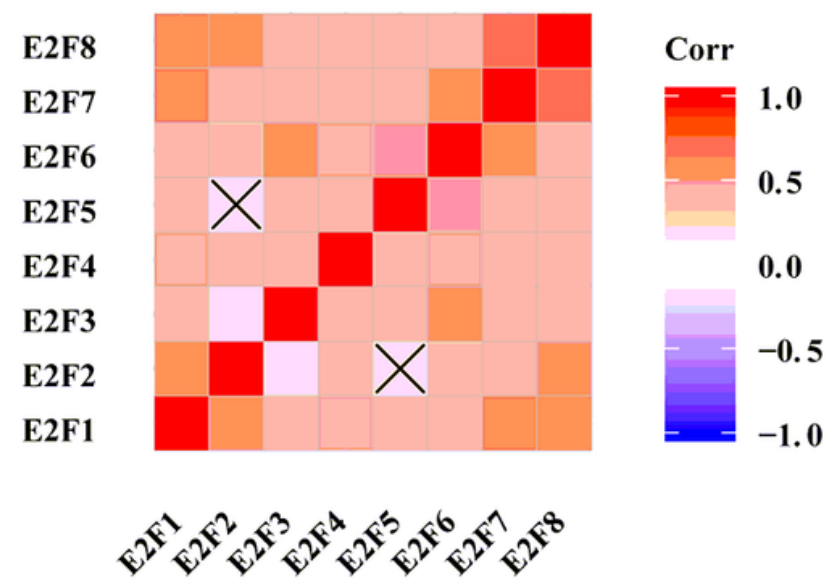

B

\section{ESCC}

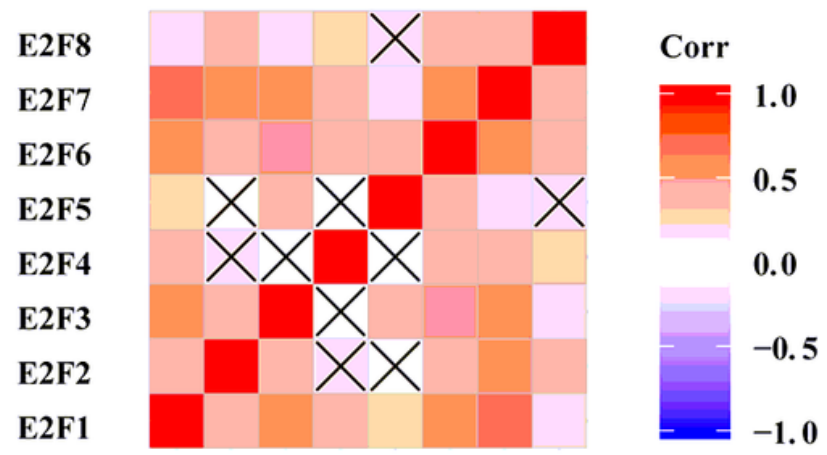

C

ESCA

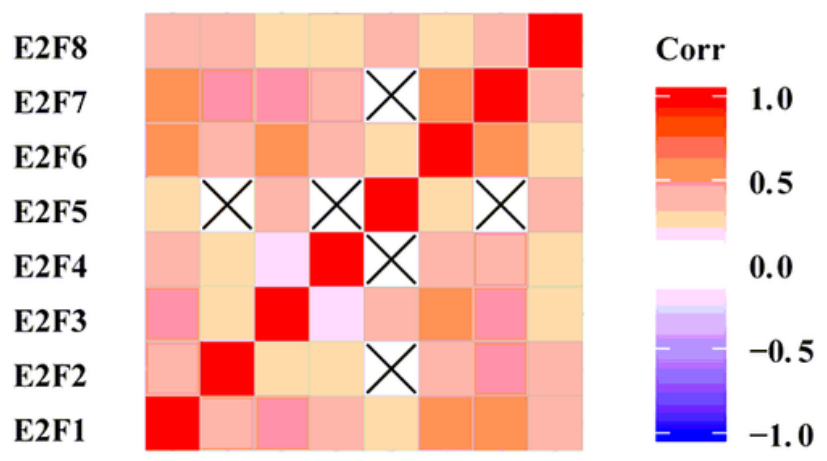

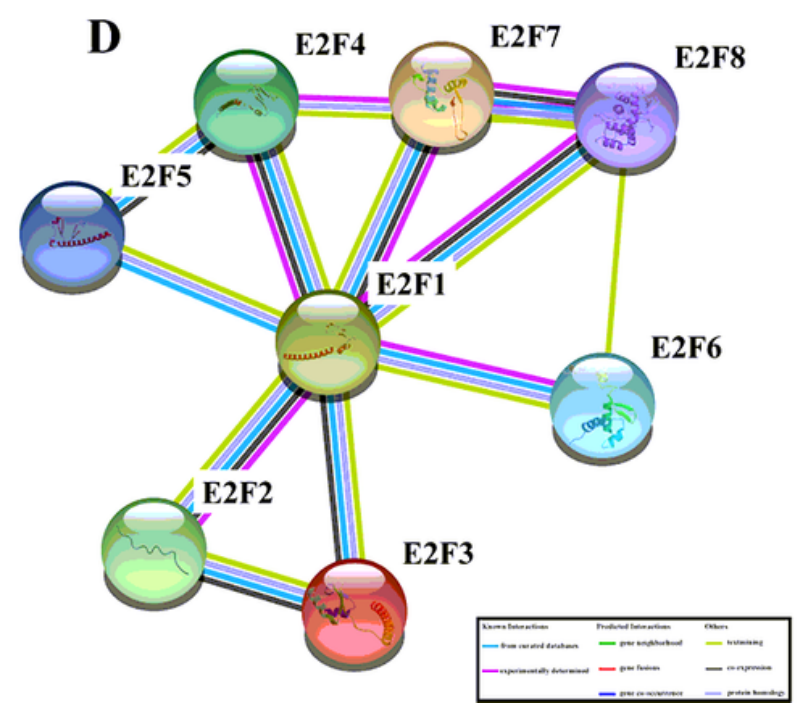

\section{Figure 4}

Coexpression and interactions between the E2F family members. (A-C) The correlation heatmap demonstrating the correlation between E2F family members in ESCC and EAC. Significant value: $P<0.05$; (D) Protein-protein interactions between E2Fs. The STRING database was used to construct the proteinprotein interactions among E2Fs. Red represents a positive correlation, blue represents a negative correlation, and X represents a nonsignificant difference. ESCA: esophageal carcinoma; ESCC: esophageal squamous cell carcinoma; EAC: esophageal adenocarcinoma; ESCA: esophageal carcinoma. 
A

EAC

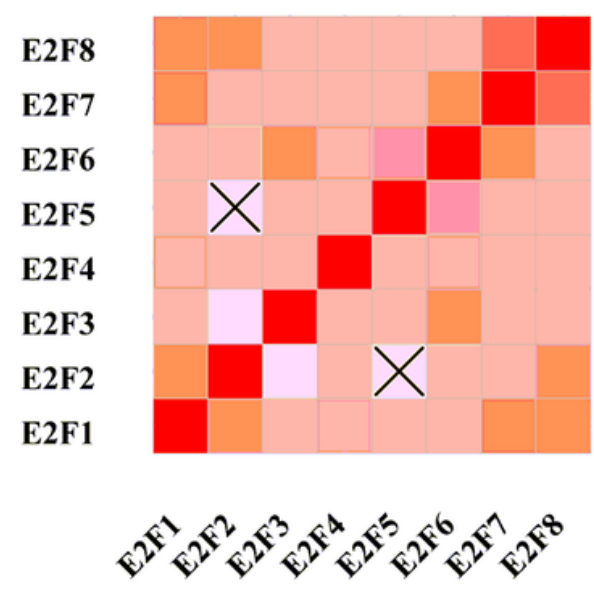

C

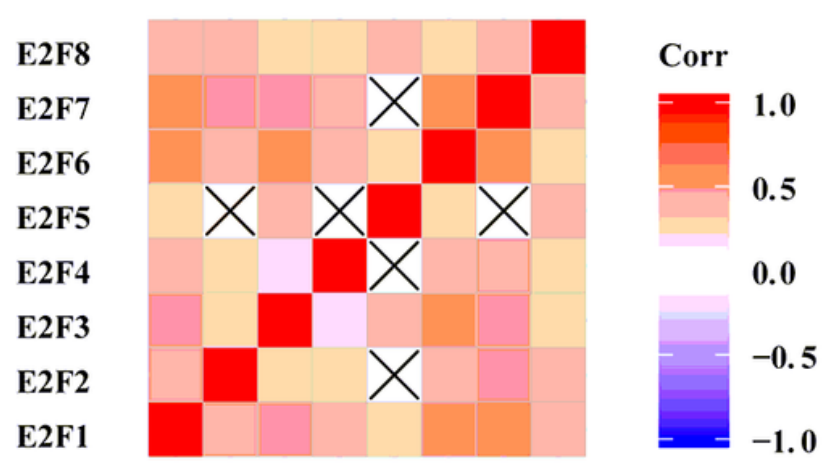

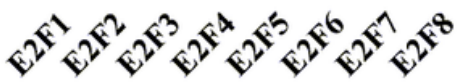

B

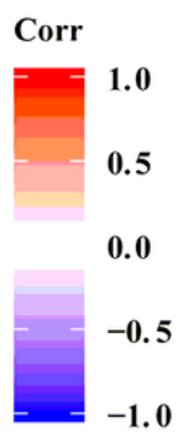

E2F8
E2F7
E2F6
E2F5
E2F4
E2F3
E2F2
E2F1

ESCC

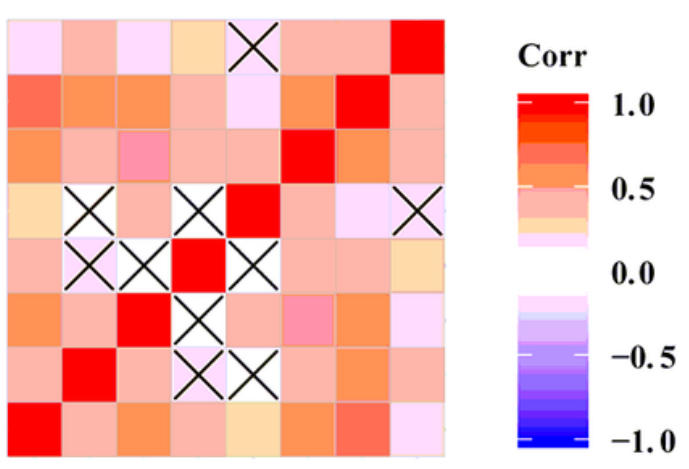

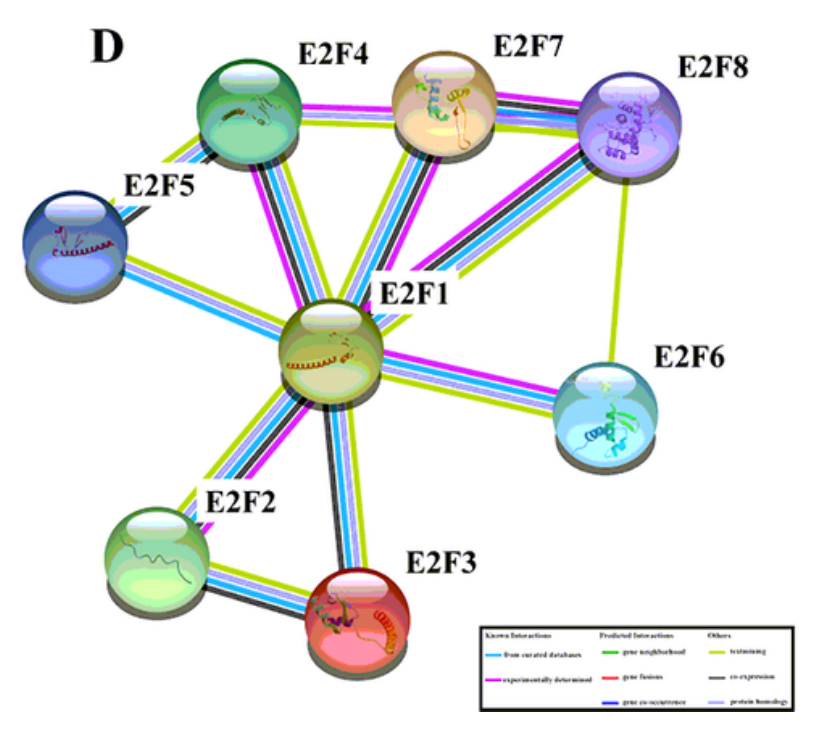

Figure 4

Coexpression and interactions between the E2F family members. (A-C) The correlation heatmap demonstrating the correlation between E2F family members in ESCC and EAC. Significant value: $P<0.05$; (D) Protein-protein interactions between E2Fs. The STRING database was used to construct the proteinprotein interactions among E2Fs. Red represents a positive correlation, blue represents a negative correlation, and X represents a nonsignificant difference. ESCA: esophageal carcinoma; ESCC: esophageal squamous cell carcinoma; EAC: esophageal adenocarcinoma; ESCA: esophageal carcinoma. 


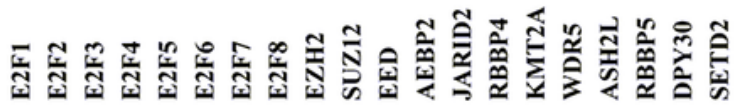

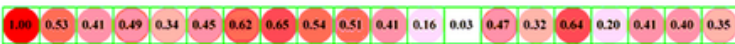

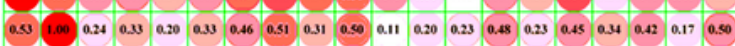

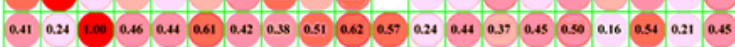

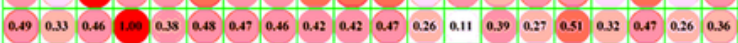

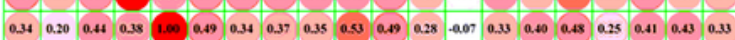

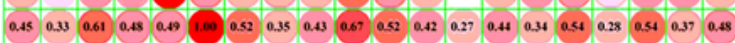

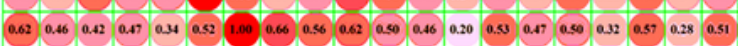

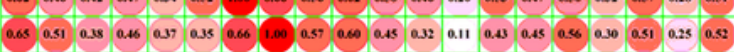

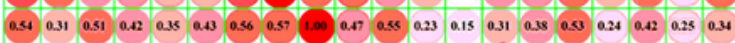

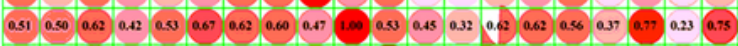

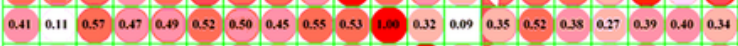
$\begin{array}{lllllllllllllllllllllllllll}0.16 & 0.20 & 0.24 & 0.26 & 0.28 & 0.42 & 0.46 & 0.32 & 0.23 & 0.45 & 0.32 & 1.00 & 0.30 & 0.26 & 0.45 & 0.21 & 0.26 & 0.43 & 0.16 & 0.45\end{array}$ \begin{tabular}{llllllllllllllllllllll}
\hline 0.03 & 0.23 & 0.44 & 0.11 & -0.07 & 0.27 & 0.20 & 0.11 & 0.15 & 0.32 & 0.05 & 0.30 & 1.00 & 0.18 & 0.26 & 0.06 & 0.14 & 0.27 & -0.11 & 0.43
\end{tabular}

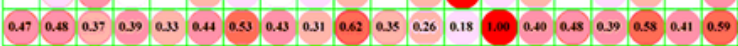

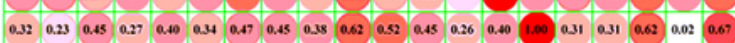

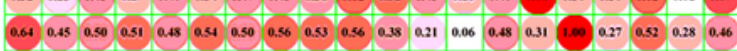

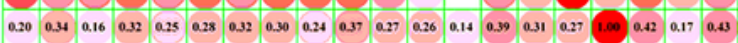

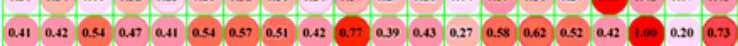

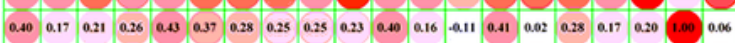

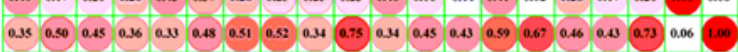

ESCA

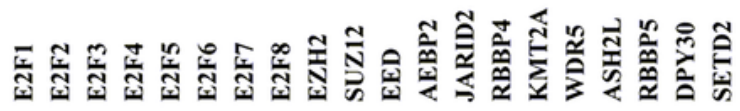

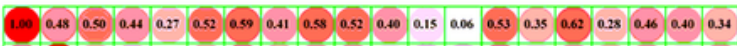

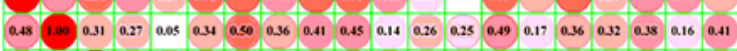

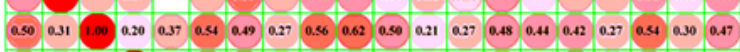

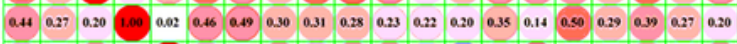

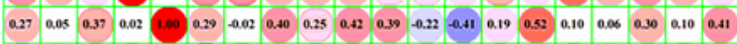

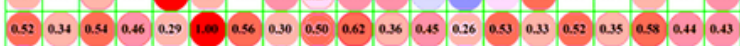

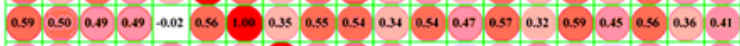

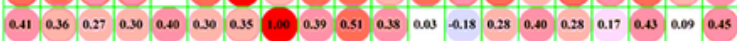

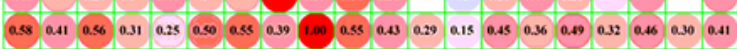

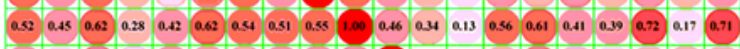

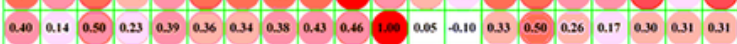

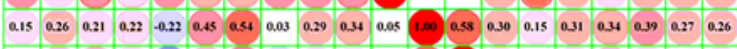

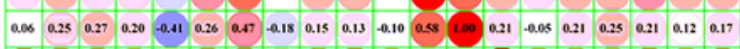

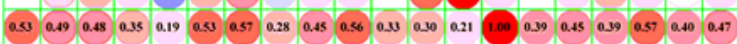

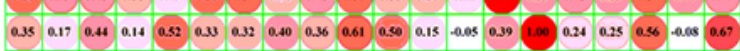

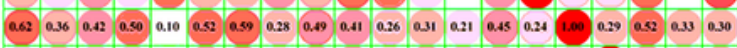

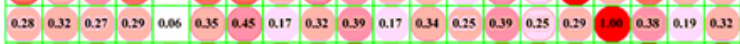

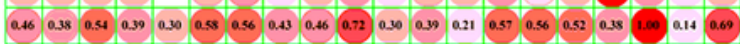

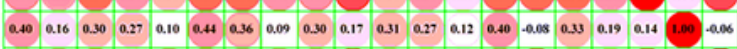

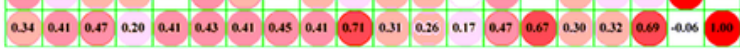

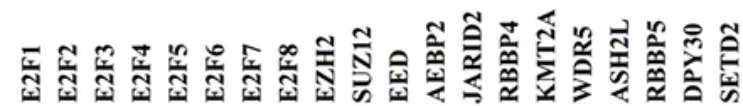

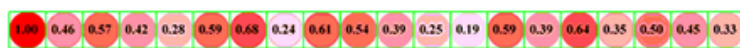

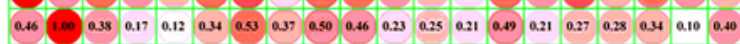

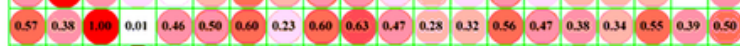

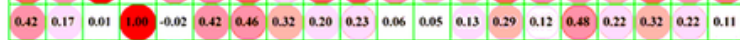

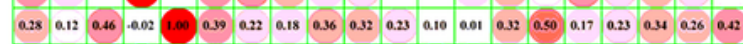

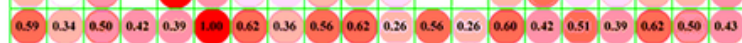

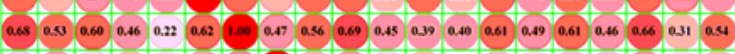

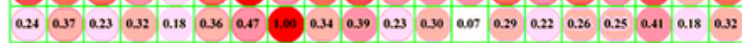

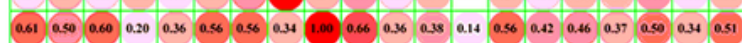

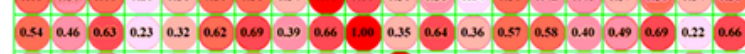

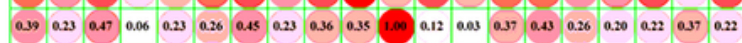

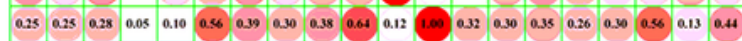

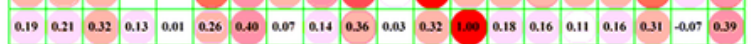

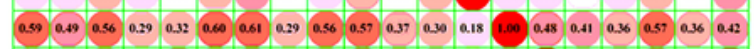

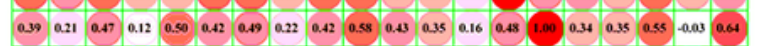

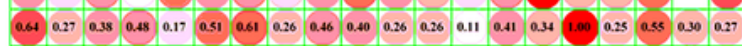

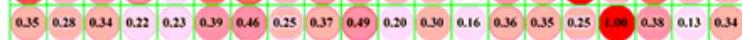

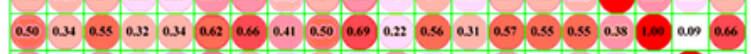

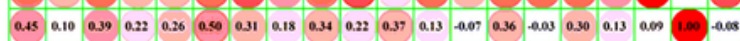

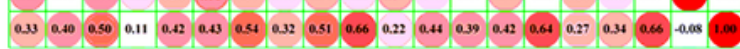

B

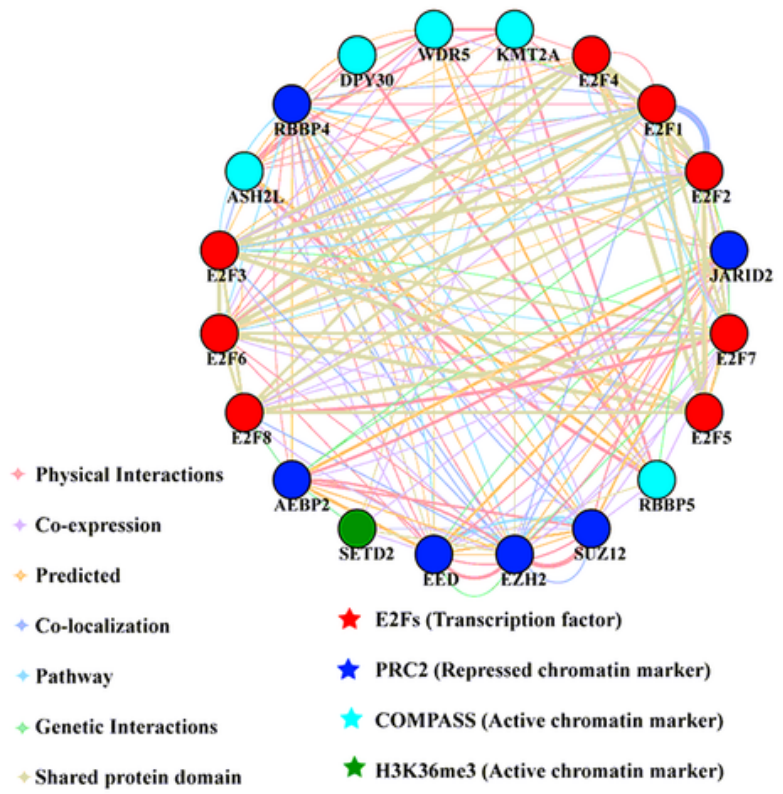

\section{Figure 5}

Interactions of the E2F family members with proteins involved in the m6A methylation pathway. Expression correlation (A) and protein-protein interaction network $(B)$ between E2Fs and N6methyladenosine ( $\mathrm{m} 6 \mathrm{~A}$ ) regulators. ESCA: esophageal carcinoma; ESCC: esophageal squamouscell carcinoma; EAC: esophageal adenocarcinoma; ESCA: esophageal carcinoma. 


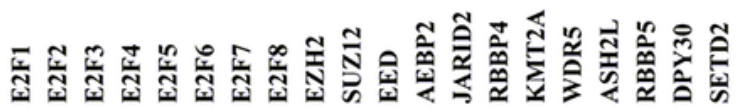

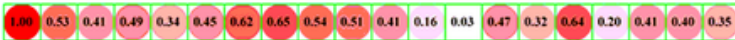

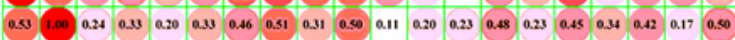

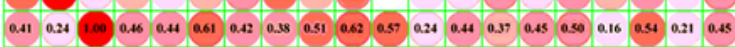

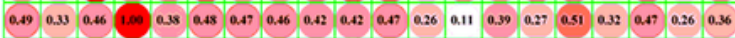

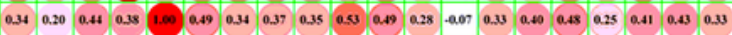

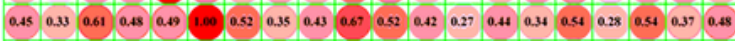
$\begin{array}{llllllllllllllllllll}0.62 & 0.46 & 0.42 & 0.47 & 0.34 & 0.52 & 1.00 & 0.66 & 0.56 & 0.62 & 0.50 & 0.46 & 0.20 & 0.50 & 0.47 & 0.50 & 0.32 & 0.57 & 0.28 & 0.51\end{array}$

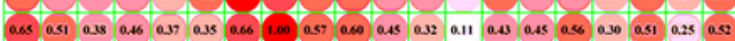

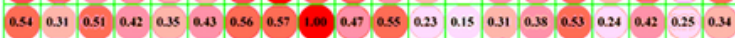

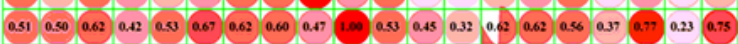

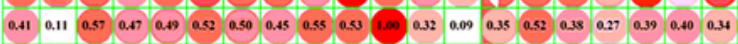

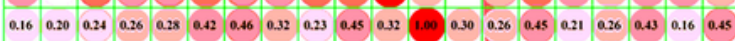
\begin{tabular}{lllllllllllllllllllll}
\hline 0.03 & 0.23 & 0.44 & 0.11 & -0.07 & 0.27 & 0.20 & 0.11 & 0.15 & 0.52 & 0.09 & 0.30 & 1.00 & 0.18 & 0.26 & 0.06 & 0.14 & 0.27 & -0.11 & 0.43
\end{tabular}

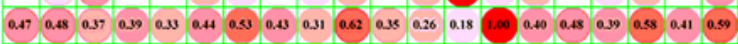

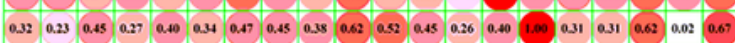

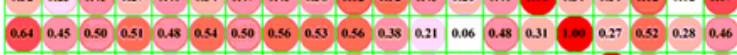

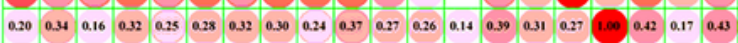

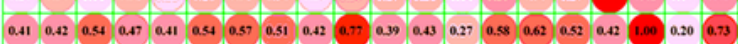
$\begin{array}{llllllllllllllllllll}0.40 & 0.17 & 0.21 & 0.26 & 0.43 & 0.37 & 0.28 & 0.25 & 0.25 & 0.23 & 0.40 & 0.16 & -0.11 & 0.41 & 0.02 & 0.28 & 0.17 & 0.20 & 1.00 & 0.06\end{array}$

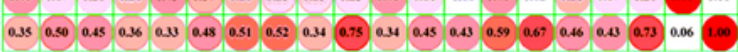

ESCA

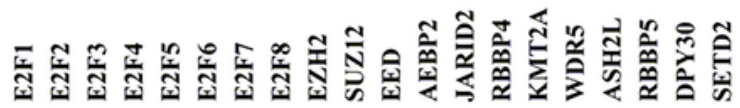

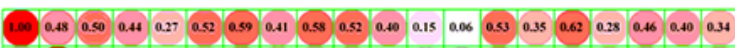

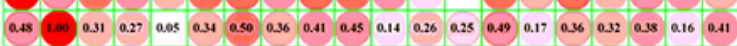

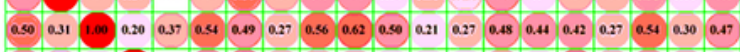

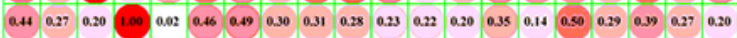

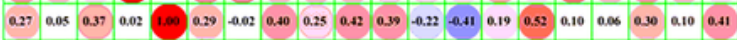

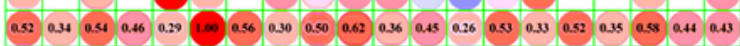

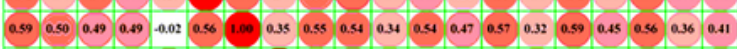

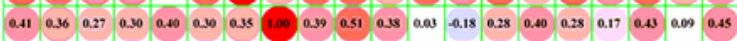

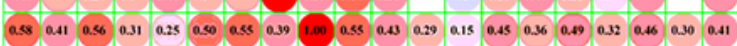

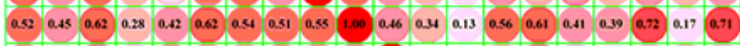

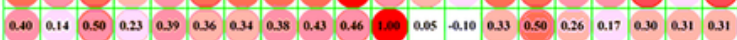

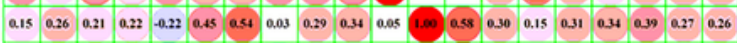

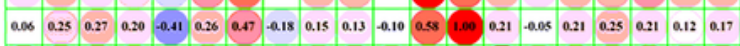

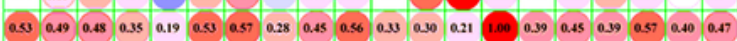

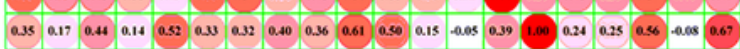

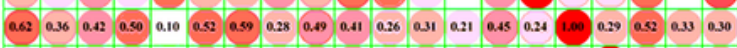

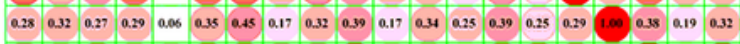

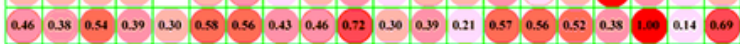

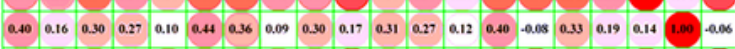

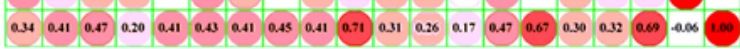

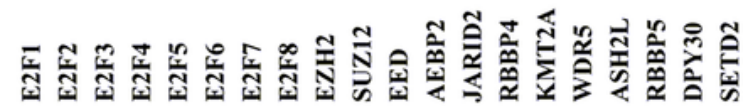

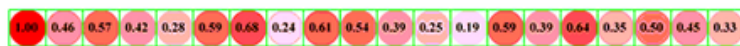

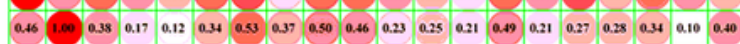

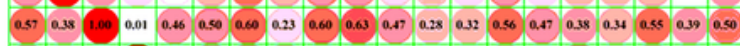

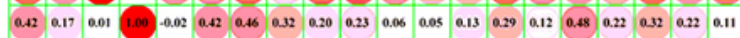

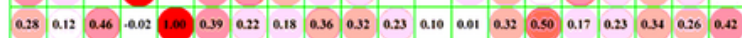

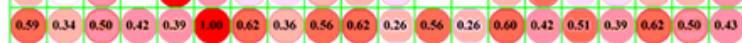

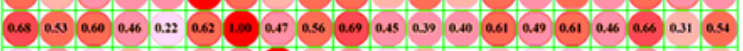

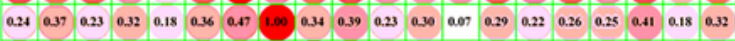

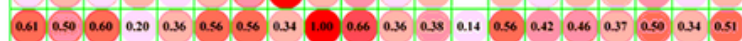

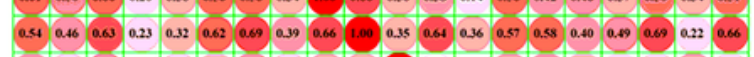

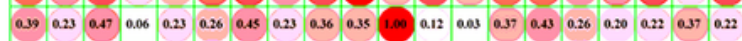

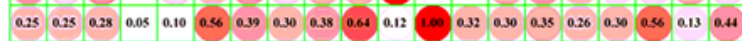

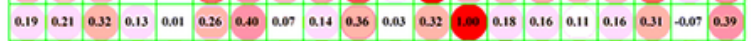

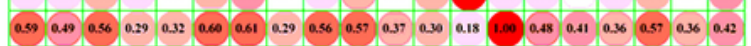

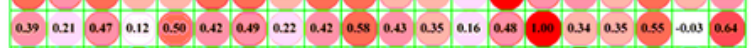

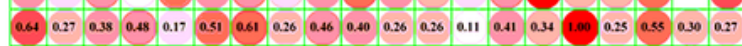

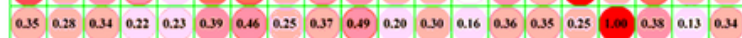

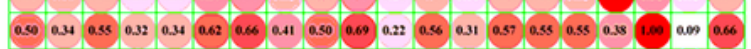

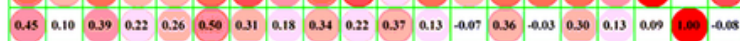

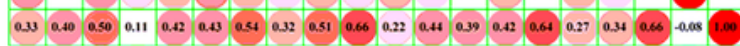

B

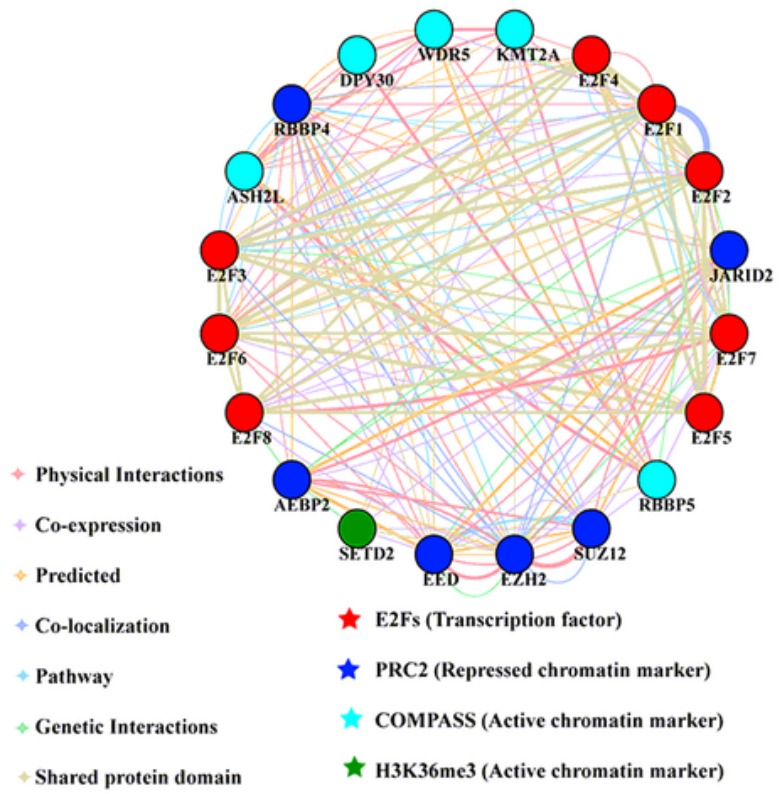

\section{Figure 5}

Interactions of the E2F family members with proteins involved in the m6A methylation pathway. Expression correlation (A) and protein-protein interaction network (B) between E2Fs and N6methyladenosine (m6A) regulators. ESCA: esophageal carcinoma; ESCC: esophageal squamouscell carcinoma; EAC: esophageal adenocarcinoma; ESCA: esophageal carcinoma. 


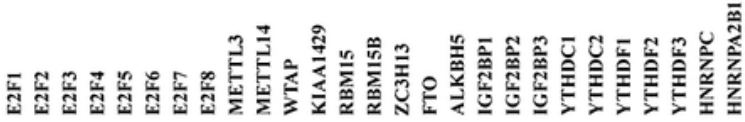

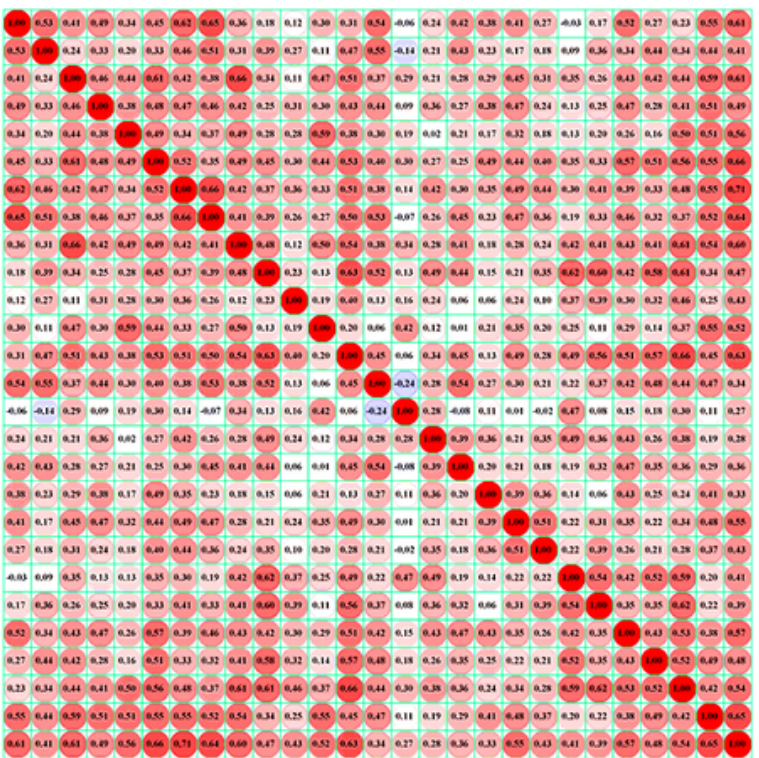

EASC

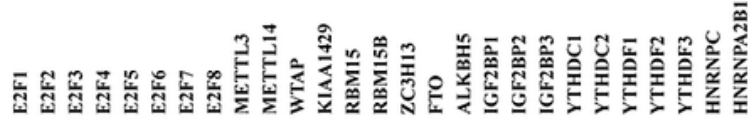

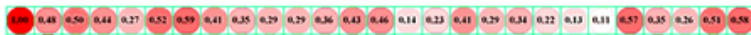

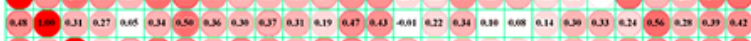

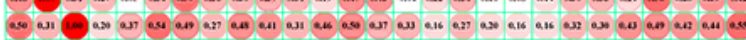

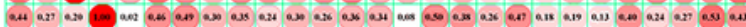

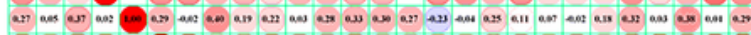

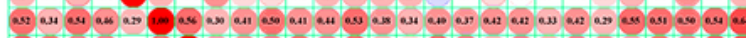

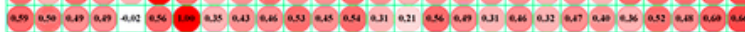

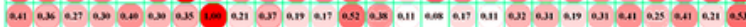

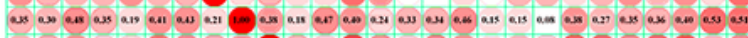

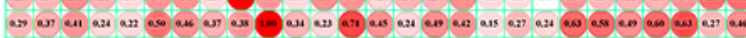

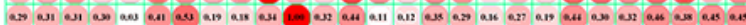

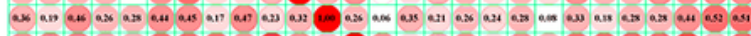

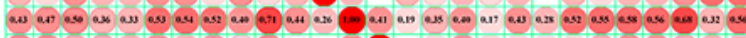

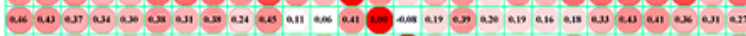

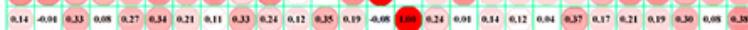

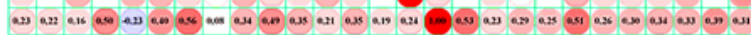

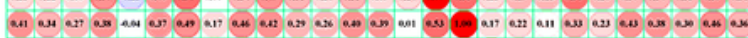

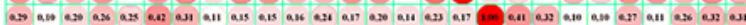

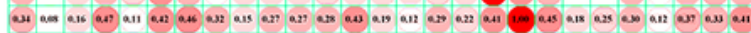

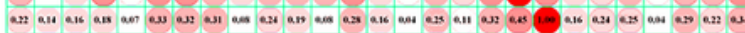

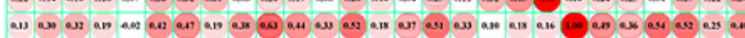

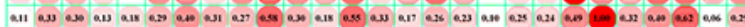

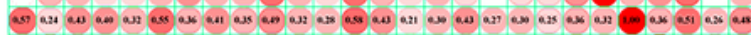

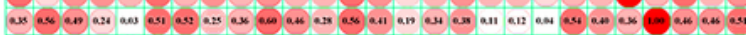

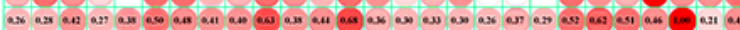

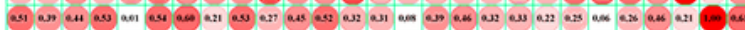

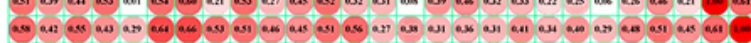

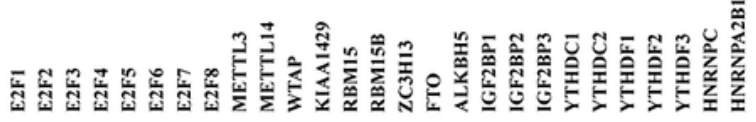

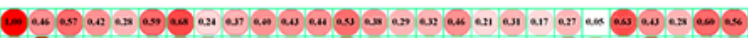

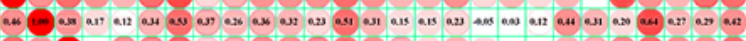

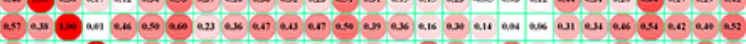

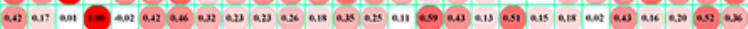

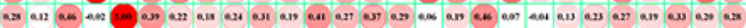

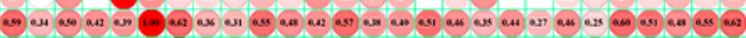

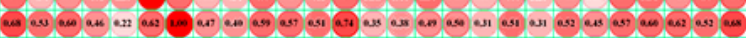

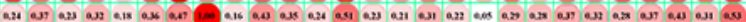

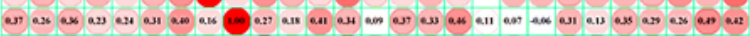

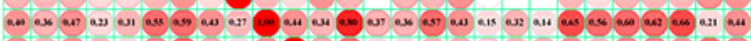

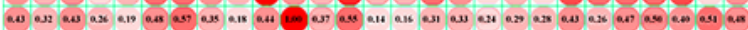

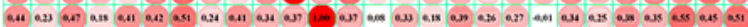

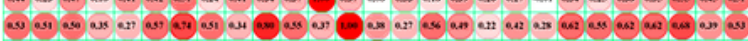

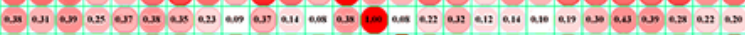

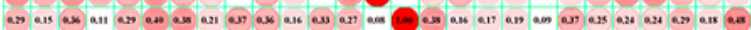

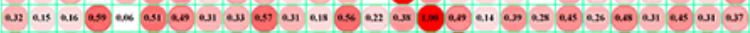

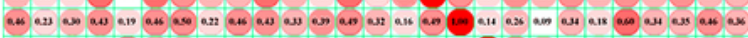

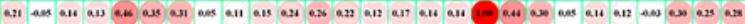

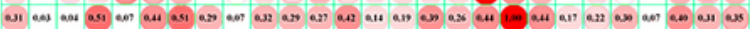

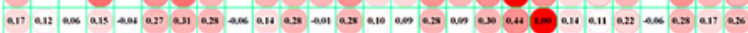

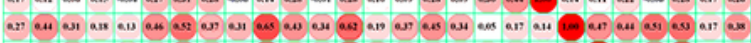

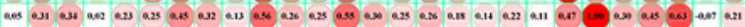

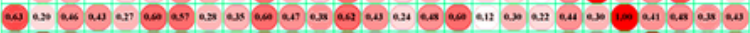

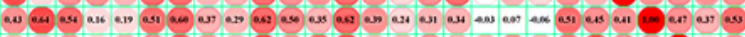

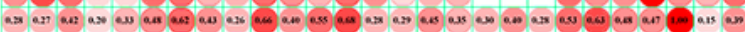

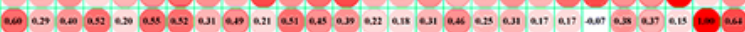

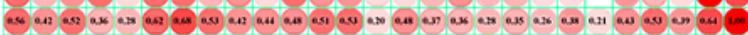

B

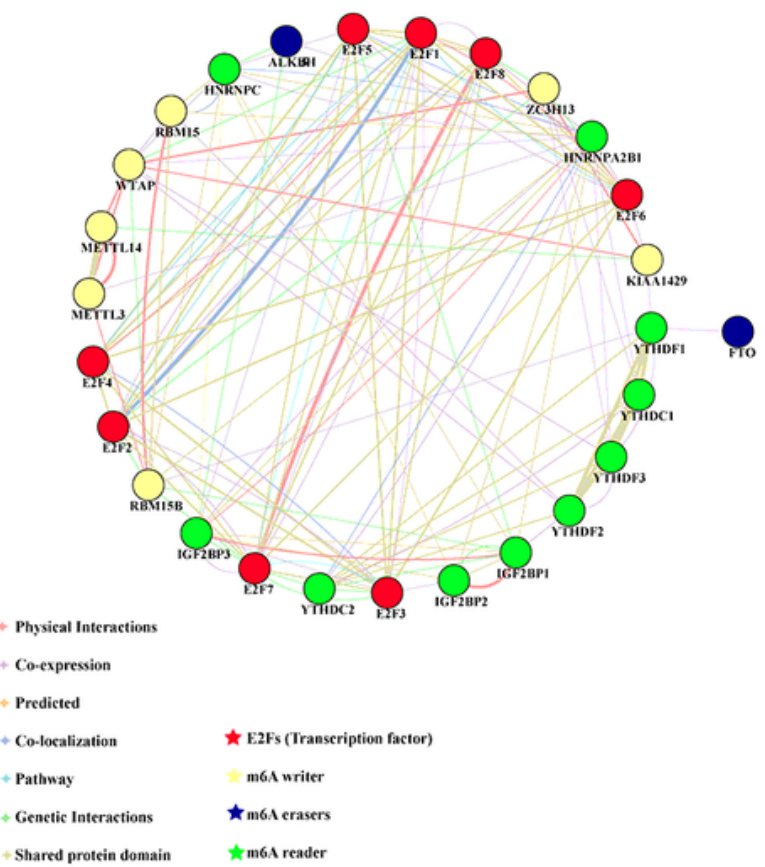

Figure 6

Interactions of the E2F family members with proteins involved in histone modifications. Expression correlation (A) and the protein-protein interaction network (B) among E2Fs and histone modification regulators. ESCA: esophageal carcinoma; ESCC: esophageal squamouscell carcinoma; EAC: esophageal adenocarcinoma; ESCA: esophageal carcinoma. 


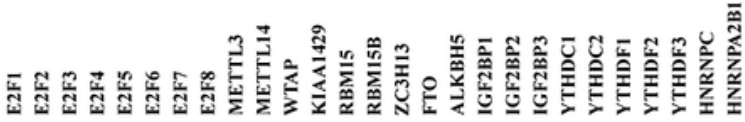

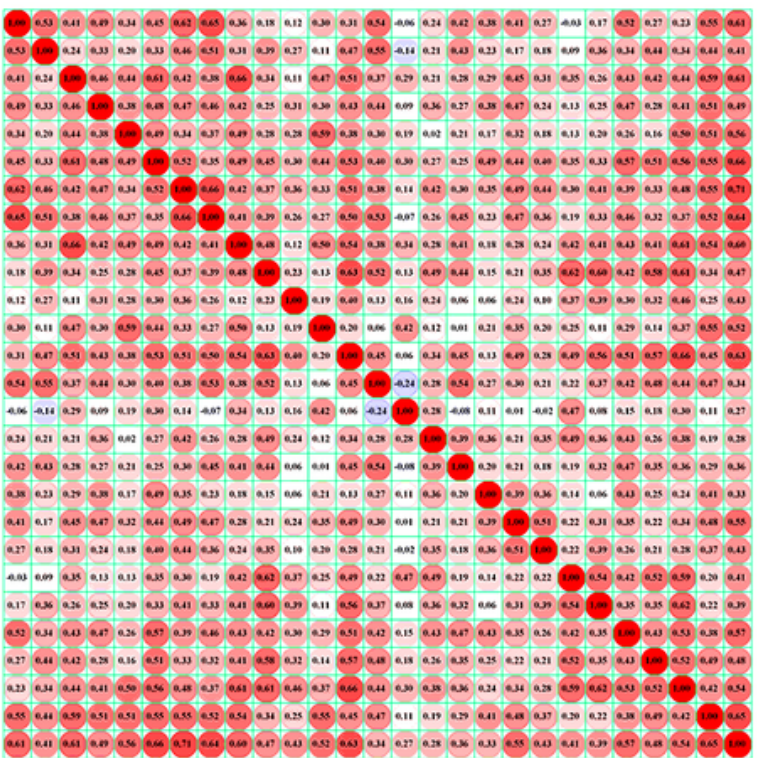

EASC

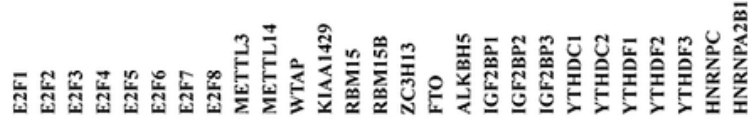

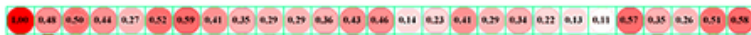

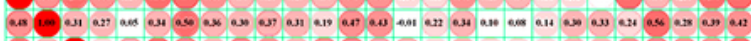

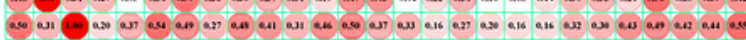

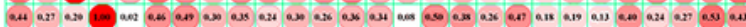

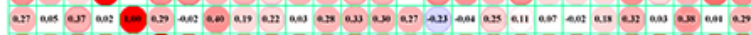

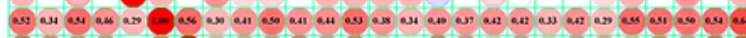

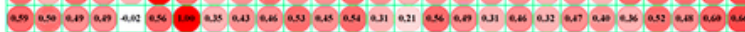

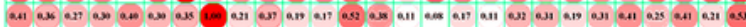

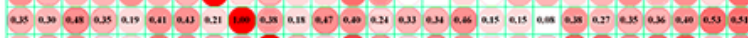

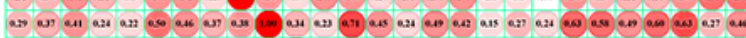

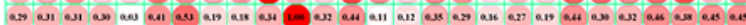

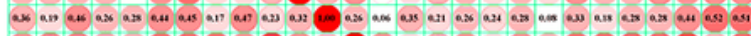

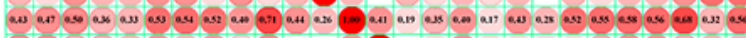

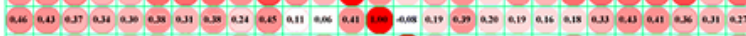

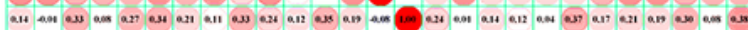

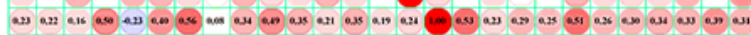

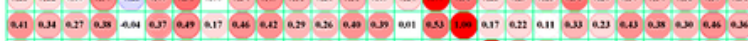

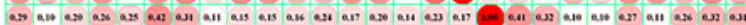

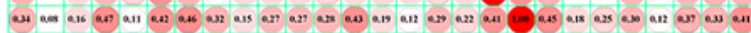

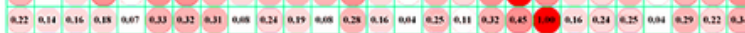

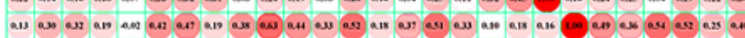

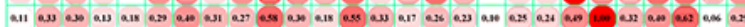

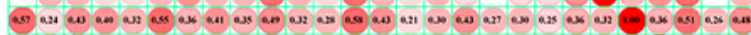

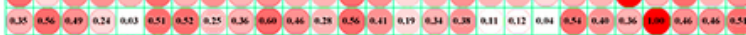

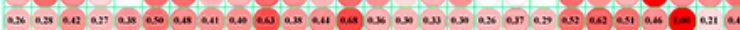

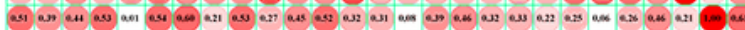

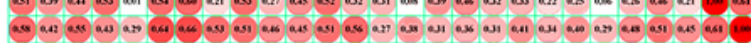

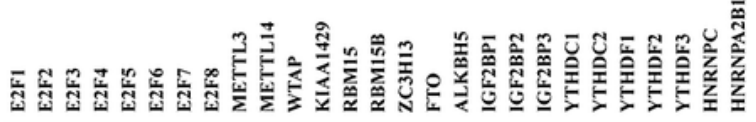

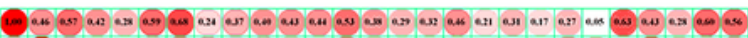

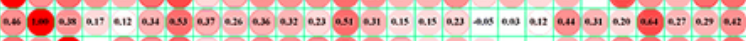

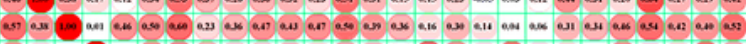

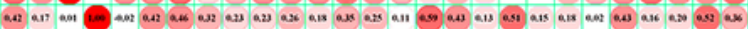

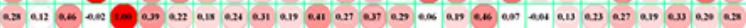

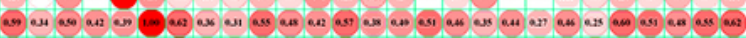

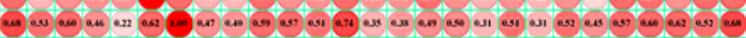

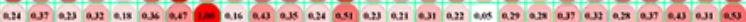

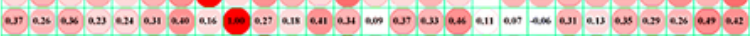

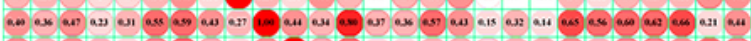

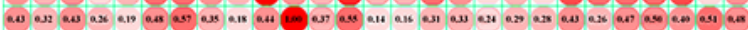

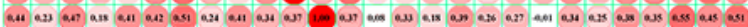

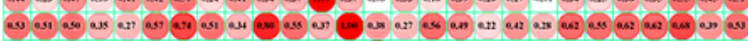

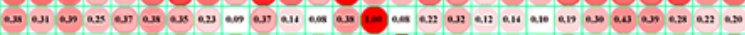

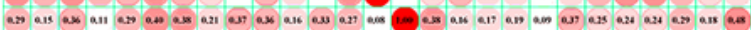

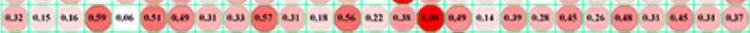

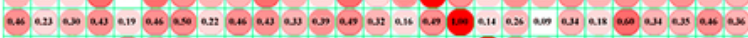

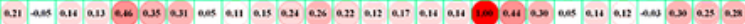

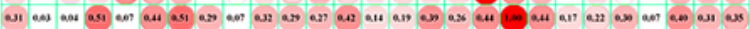

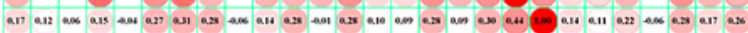

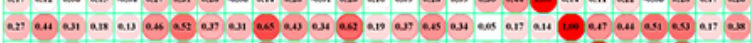

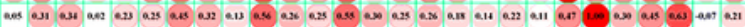

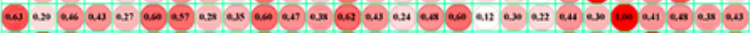

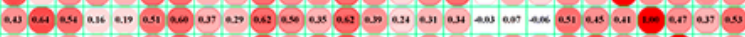

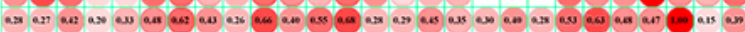

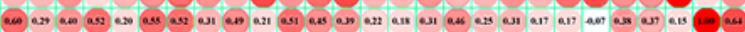

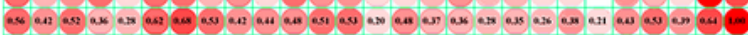

B

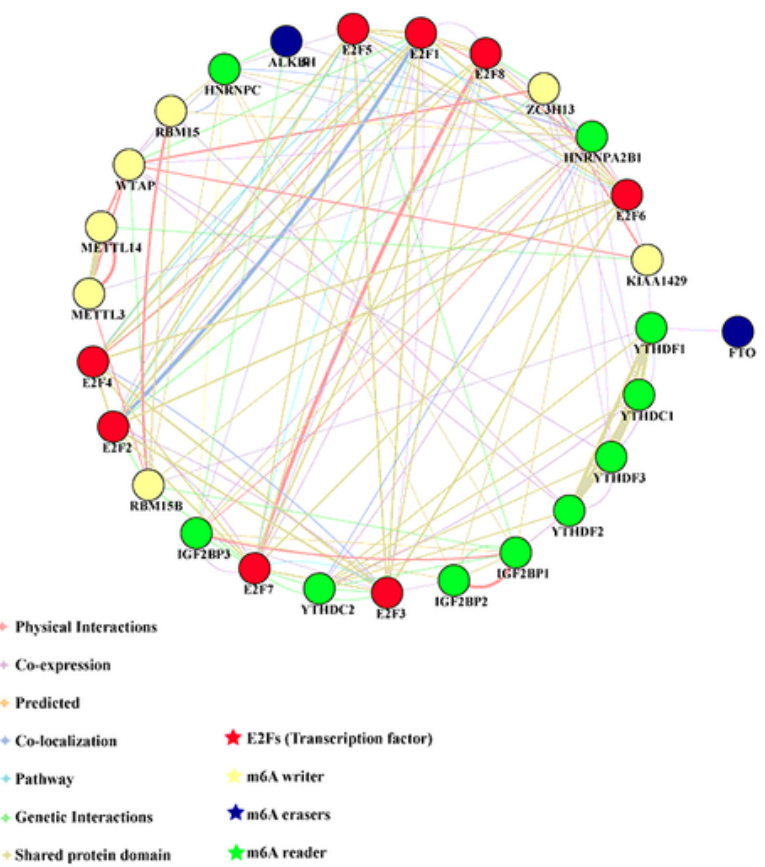

Figure 6

Interactions of the E2F family members with proteins involved in histone modifications. Expression correlation (A) and the protein-protein interaction network (B) among E2Fs and histone modification regulators. ESCA: esophageal carcinoma; ESCC: esophageal squamouscell carcinoma; EAC: esophageal adenocarcinoma; ESCA: esophageal carcinoma. 


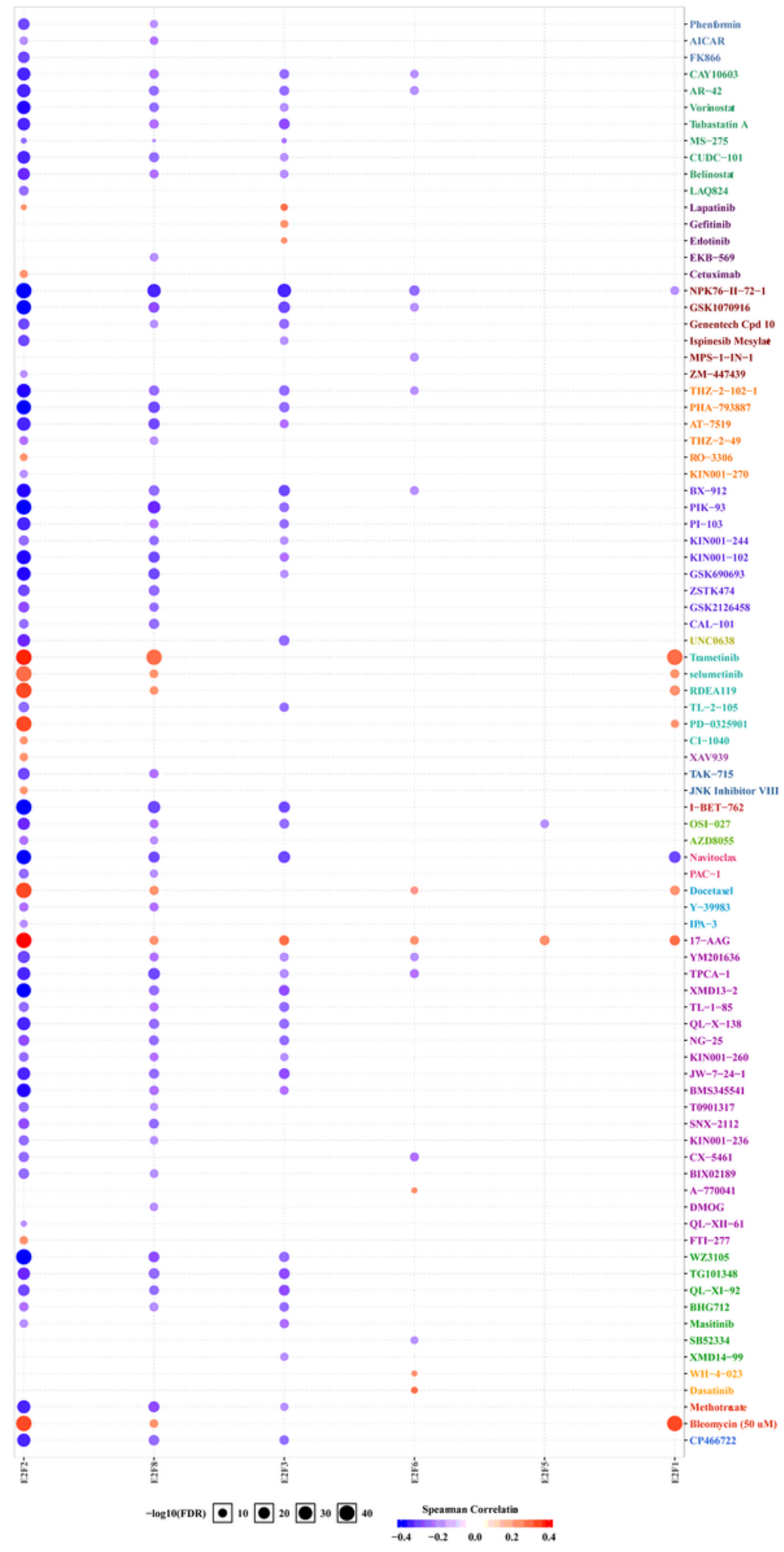

Figure 7

Correlation between E2Fs and anticancer drug resistance to common drugs. The bioinformatics database GSCA Lite was used to calculate and visualize the correlation between E2Fs and common anticancer drugs. The circle size represents the correlation strength, red represents a positive correlation, and blue represents a negative correlation. A false discovery rate $<0.05$ indicated a statistical significance. 


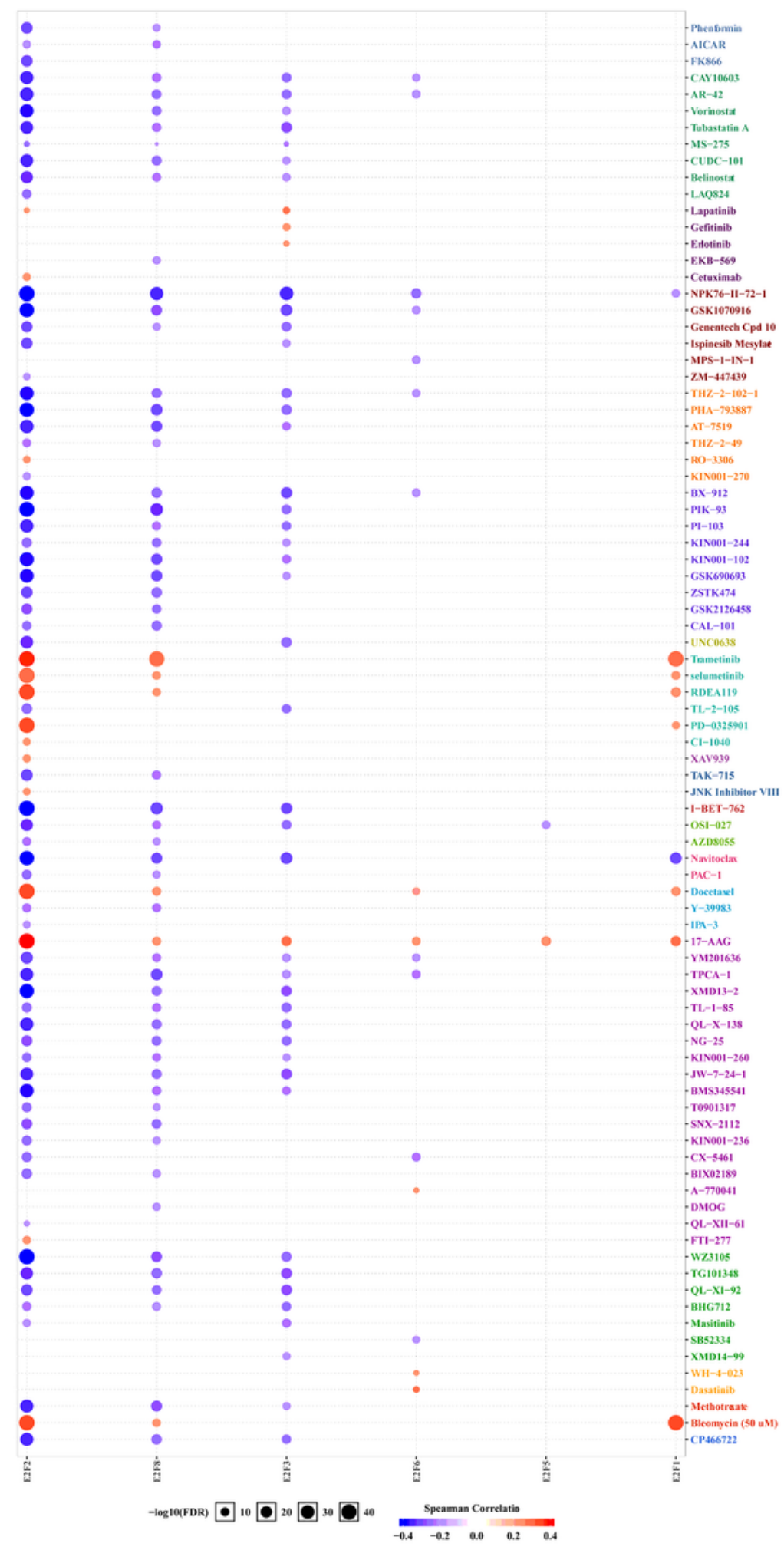

\section{Figure 7}

Correlation between E2Fs and anticancer drug resistance to common drugs. The bioinformatics database GSCA Lite was used to calculate and visualize the correlation between E2Fs and common anticancer drugs. The circle size represents the correlation strength, red represents a positive correlation, and blue represents a negative correlation. A false discovery rate 0.05 indicated a statistical significance. 
A
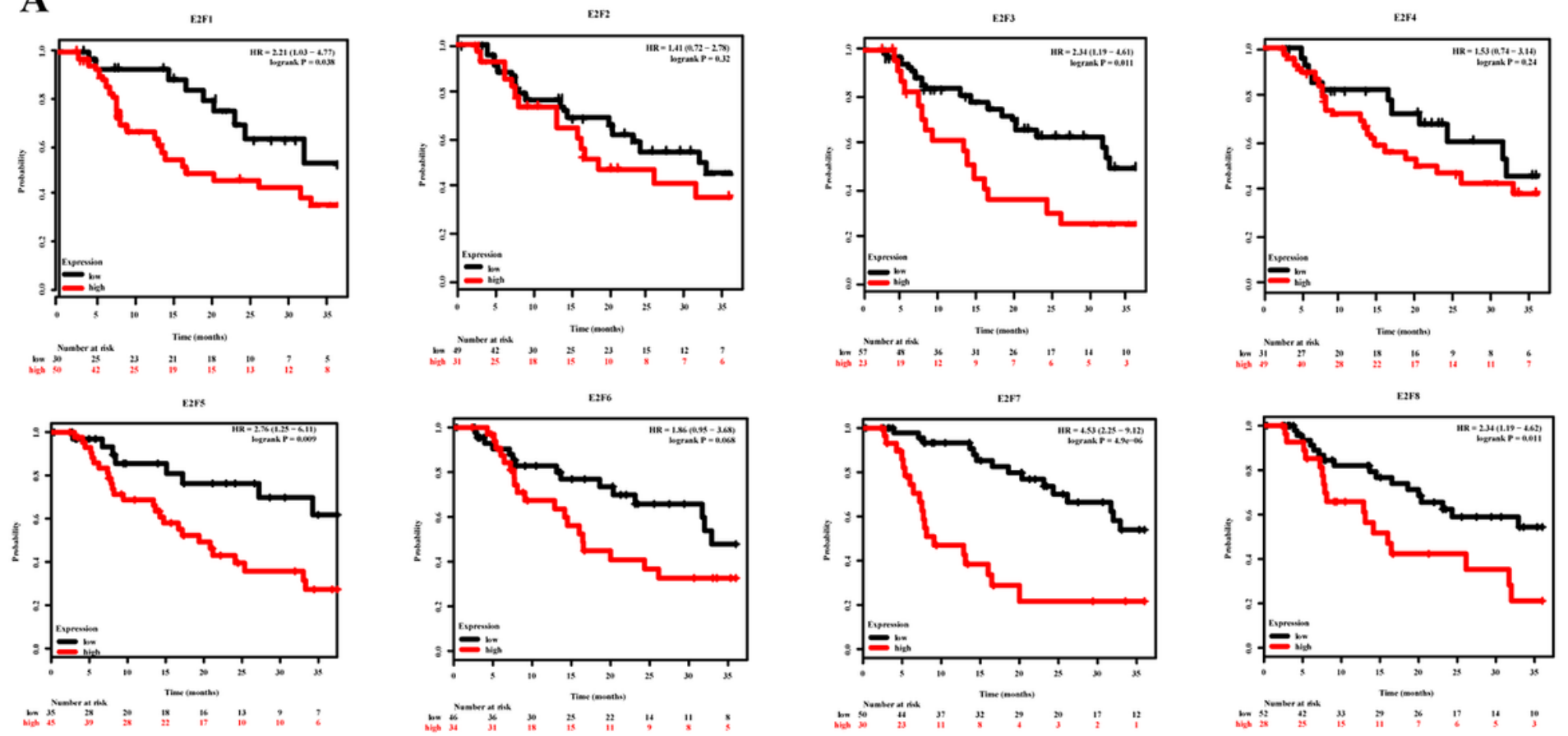

B
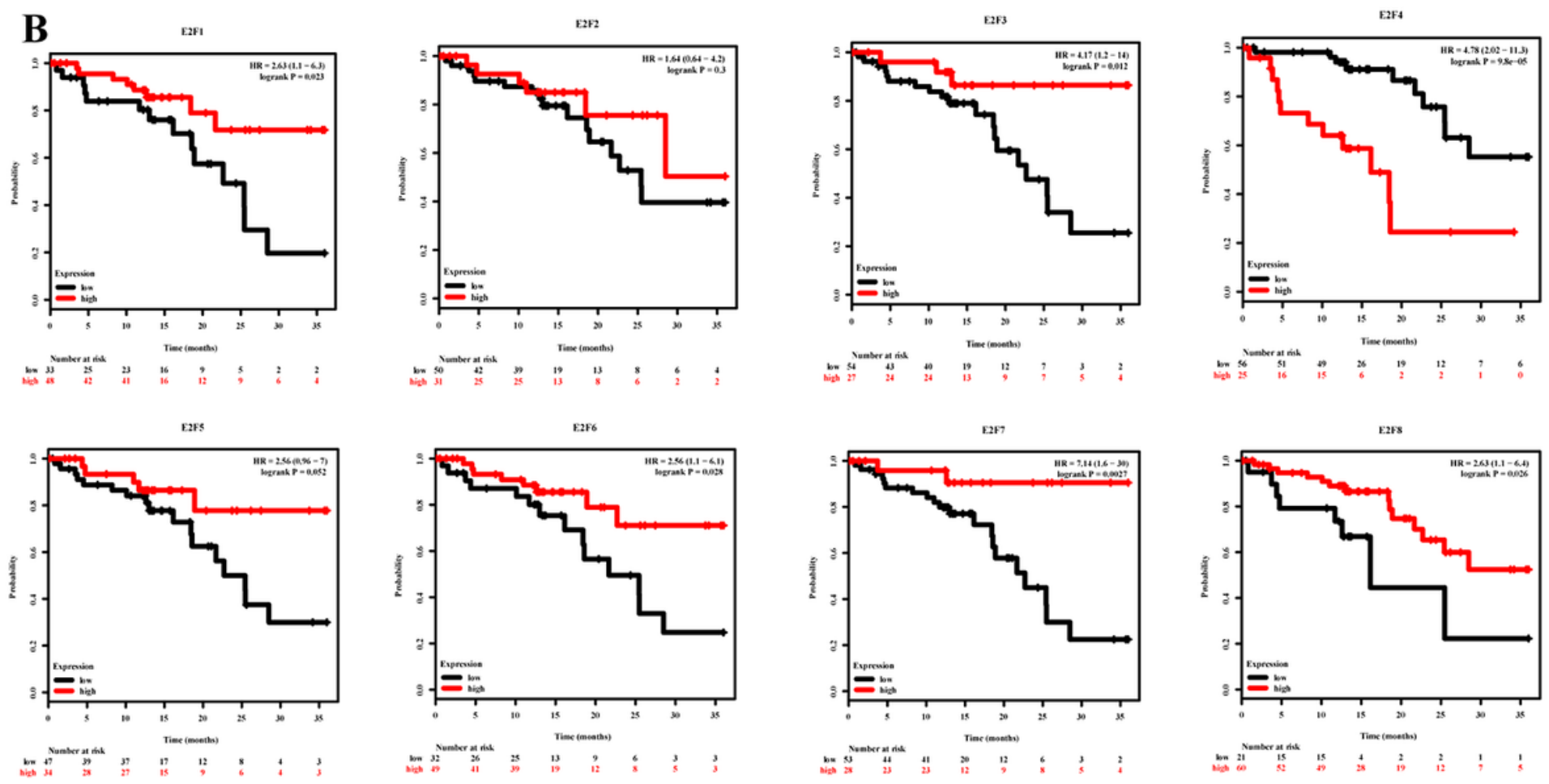

\section{Figure 8}

Kaplan-Meier Plotter analysis of the correlation between the mRNA expression of E2Fs and the prognosis of patients with ESCC or EAC. (A) The prognostic value (overall survival, OS) of the mRNA level of E2Fs in EAC (Kaplan-Meier Plotter). (B) The prognostic value (OS) of the mRNA level of E2Fs in ESCC (Kaplan-Meier Plotter). A P-value 0.05 indicated a statistically significant difference. 
A
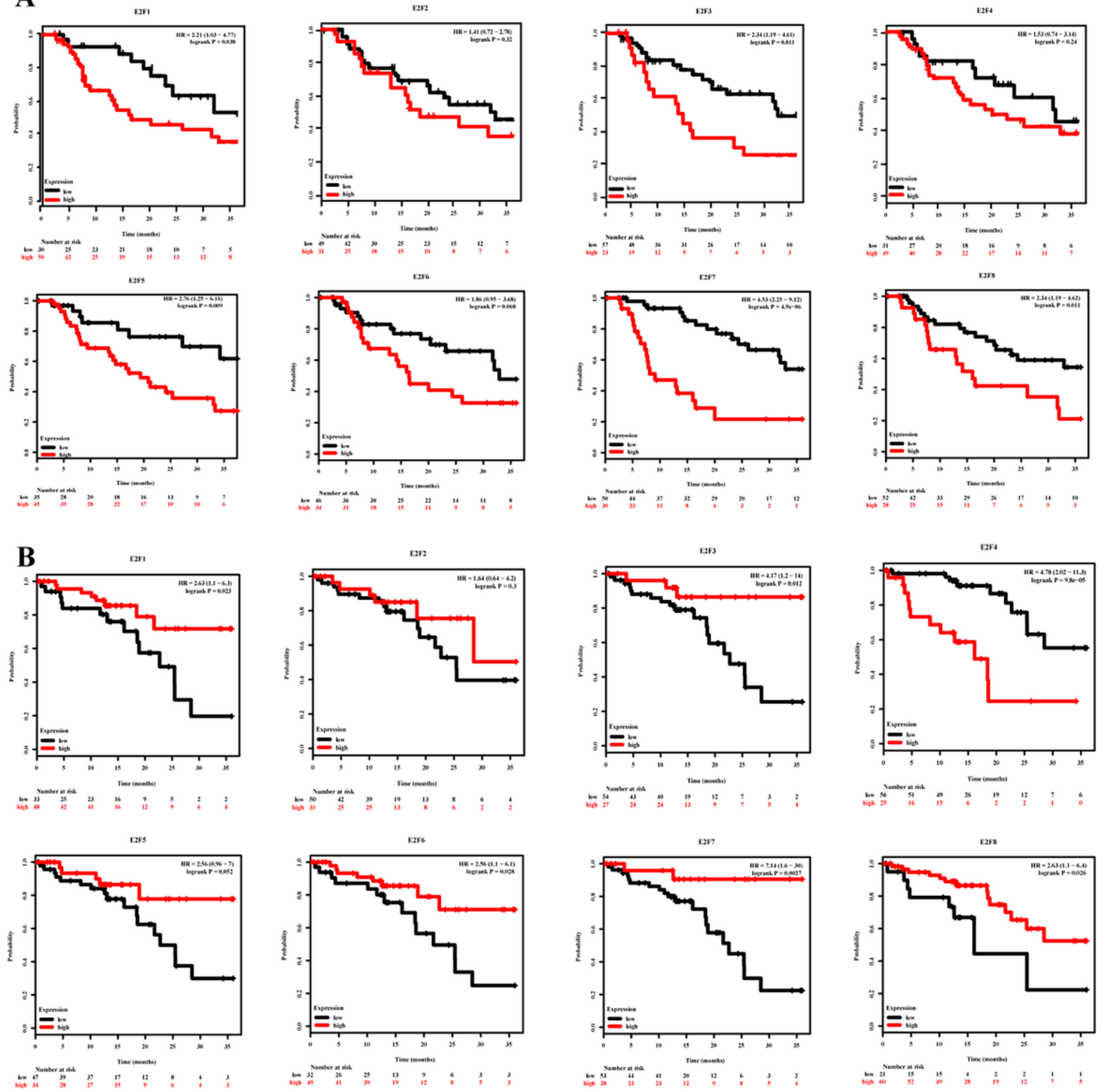

\section{Figure 8}

Kaplan-Meier Plotter analysis of the correlation between the mRNA expression of E2Fs and the prognosis of patients with ESCC or EAC. (A) The prognostic value (overall survival, OS) of the mRNA level of E2Fs in EAC (Kaplan-Meier Plotter). (B) The prognostic value (OS) of the mRNA level of E2Fs in ESCC (Kaplan-Meier Plotter). A P-value< 0.05 indicated a statistically significant difference. 